\title{
Deep asteroseismic sounding of the compact hot B subdwarf pulsator KIC02697388 from Kepler time series photometry ${ }^{\star}$
}

\author{
S. Charpinet ${ }^{1,2}$, V. Van Grootel ${ }^{3, \star \star}$, G. Fontaine ${ }^{4}$, E. M. Green ${ }^{5}$, P. Brassard ${ }^{4}$, S. K. Randall ${ }^{6}$, R. Silvotti ${ }^{7}$ \\ R. H. Østensen ${ }^{8}$, H. Kjeldsen ${ }^{9}$, J. Christensen-Dalsgaard ${ }^{9}$, S. D. Kawaler ${ }^{10}$, B. D. Clarke ${ }^{11}$, J. Li ${ }^{11}$, and B. Wohler ${ }^{12}$ \\ 1 Université de Toulouse, UPS-OMP, IRAP, Toulouse, France \\ 2 CNRS, IRAP, 14 avenue Edouard Belin, 31400 Toulouse, France \\ e-mail: stephane. charpinet@ast.obs-mip.fr \\ 3 Institut d'Astrophysique et de Géophysique, Université de Liège, 17 Allée du 6 Août, 4000 Liège, Belgium \\ e-mail: vangroot@astro.ulg.ac.be \\ ${ }^{4}$ Département de Physique, Université de Montréal, CP 6128, Succursale Centre-Ville, Montréal, QC H3C 3J7, Canada \\ e-mail: [fontaine; brassard] @astro.umontreal.ca \\ 5 Steward Observatory, University of Arizona, 933 North Cherry Avenue, Tucson, AZ 85721, USA \\ e-mail: egreen@email.arizona.edu \\ ${ }^{6}$ ESO, Karl-Schwarzschild-Str. 2, 85748 Garching bei München, Germany \\ e-mail: srandall@eso.org \\ 7 INAF-Osservatorio Astronomico di Torino, Strada dell'Osservatorio 20, 10025 Pino Torinese, Italy \\ 8 Instituut voor Sterrenkunde, KU Leuven, Celestijnenlaan 200D, 3001 Leuven, Belgium \\ 9 Department of Physics and Astronomy, Aarhus University, 8000 Aarhus C, Denmark \\ 10 Department of Physics and Astronomy, Iowa State University, Ames, IA 50011, USA \\ 11 SETI Institute/NASA Ames Research Center, Moffett Field, CA 94035, USA \\ 12 Orbital Sciences Corporation/NASA Ames Research Center, Moffett Field, CA 94035, USA
}

Received 28 December 2010 / Accepted 7 March 2011

\begin{abstract}
Context. Contemporary high precision photometry from space provided by the Kepler and CoRoT satellites generates significant breakthroughs in terms of exploiting the long-period, g-mode pulsating hot B subdwarf ( $\mathrm{sdBV}_{\mathrm{s}}$ ) stars with asteroseismology. Aims. We present a detailed asteroseismic study of the $\mathrm{sdBV}_{\mathrm{s}}$ star KIC02697388 monitored with Kepler, using the rich pulsation spectrum uncovered during the $\sim 27$-day-long exploratory run Q2.3.

Methods. We analyse new high-S/N spectroscopy of KIC02697388 using appropriate NLTE model atmospheres to provide accurate atmospheric parameters for this star. We also reanalyse the Kepler light curve using standard prewhitening techniques. On this basis, we apply a forward modelling technique using our latest generation of sdB models. The simultaneous match of the independent periods observed in KIC02697388 with those of models leads objectively to the identification of the pulsation modes and, more importantly, to the determination of some of the parameters of the star.

Results. The light curve analysis reveals 43 independent frequencies that can be associated with oscillation modes. All the modulations observed in this star correspond to $g$-mode pulsations except one high-frequency signal, which is typical of a $p$-mode oscillation. Although the presence of this $p$-mode is surprising considering the atmospheric parameters that we derive for this cool sdB $\operatorname{star}\left(T_{\text {eff }}=25395 \pm 227 \mathrm{~K}, \log g=5.500 \pm 0.031(\mathrm{cgs})\right.$, and $\left.\log N(\mathrm{He}) / N(\mathrm{H})=-2.767 \pm 0.122\right)$, we show that this mode can be accounted for particularly well by our optimal seismic models, both in terms of frequency match and nonadiabatic properties. The seismic analysis leads us to identify two model solutions that can both account for the observed pulsation properties of KIC02697388. Despite this remaining ambiguity, several key parameters of the star can be derived with stringent constraints, such as its mass, its $\mathrm{H}$-rich envelope mass, its radius, and its luminosity. We derive the properties of the core proposing that it is a relatively young sdB star that has burnt less than $\sim 34 \%$ (in mass) of its central helium and has a relatively large mixed $\mathrm{He} / \mathrm{C} / \mathrm{O}$ core. This latter measurement is in line with the trend already uncovered for two other $g$-mode sdB pulsators analysed with asteroseismology and suggests that extra mixing is occurring quite early in the evolution of He cores on the horizontal branch.

Conclusions. Additional monitoring with Kepler of this particularly interesting sdB star should reveal the inner properties of KIC02697388 and provide important information about the mode driving mechanism and the helium core properties.
\end{abstract}

Key words. stars: oscillations - stars: interiors - stars: horizontal-branch - subdwarfs - stars: individual: KIC02697388

\section{Introduction}

Non-radial pulsations commonly observed in hot B subdwarf (sdB) stars offer great opportunities for sounding, by

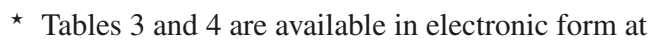
http://www . aanda.org

$\star \star$ Chargé de recherches, Fonds de la Recherche Scientifique, FNRS, rue d'Egmont 5, 1000 Bruxelles, Belgium. asteroseismic methods, the inner structure and dynamics of stars that are representative of an intermediate stage of stellar evolution. B subdwarf stars populate the so-called extreme horizontal branch $(\mathrm{EHB})$ corresponding to low-mass $\left(\sim 0.5 M_{\odot}\right)$ objects burning helium in their core (see Heber 2009, for a review of hot subdwarf stars). They differ from classical horizontal branch stars mainly in terms of their residual H-rich envelope, which has been almost entirely removed during the previous 
stage of evolution, leaving only a very thin layer less massive than $\sim 0.02 M_{\odot}$. The B subdwarfs are therefore hot and compact ( $T_{\text {eff }} \sim 22000-40000 \mathrm{~K}$ and $\log g \sim 5.2-6.2$; Saffer et al. 1994) stars that presumably never ascend the asymptotic giant branch prior to fading away as cooling white dwarfs in their subsequent evolution (e.g. Dorman et al. 1993). It remains unclear which mechanisms determine whether a star evolving through the red giant phase eventually loses (or not) all but a tiny fraction of its envelope. Compact binary evolution across various channels is probably an important source of sdB stars (Han et al. 2002, 2003). But isolated main-sequence progenitors passing through the red giant branch and experiencing enhanced mass loss (D'Cruz et al. 1996) cannot be excluded, considering that a significant fraction of sdB stars $(\sim 50 \%)$ remain apparently single objects or non-interacting binaries (Geier et al. 2009a). The merger of two helium white dwarfs has often been proposed to explain the origin of isolated sdB stars (Han et al. 2002, 2003) but one would expect these to be rapidly rotating and evidence of this is severely lacking so far (Geier et al. 2009b). An interesting idea proposed by Soker (1998) is that massive planets in close orbits ( $\$ 5 \mathrm{AU}$ ) could interact with the envelope of the expanding red giant star, transfering some of their orbital angular momentum to the envelope, speeding it up, and thus enhancing the mass loss. Considering that stars with close orbiting giant planets are fairly common, i.e., 6.6\% have planets within $5 \mathrm{AU}$ (Marcy et al. 2005; Udry \& Santos 2007), the formation of isolated hot B subdwarf stars may very well be a consequence, at least for some of them, of the presence of these planetary systems. We point out that two sdB stars show evidence of orbiting giant planets that survived the red giant branch episode: V391 Peg (Silvotti et al. 2007) and the close binary HW Vir with its two circumbinary planets (Lee et al. 2009).

Two groups of sdB pulsators offer favorable conditions for developing asteroseismology as a new tool to investigate this intermediate evolutionary stage. The $\mathrm{sdBV}_{\mathrm{r}}$ (or V361 Hya, or EC 14026; Kilkenny et al. 1997) stars oscillate with periods in the 100-600 s range, corresponding mostly to low-order, low-degree $p$-modes. These modes are driven by a $\kappa$-mechanism induced by the M-shell ionization of iron-group elements (the so-called $Z$-bump in the mean Rosseland opacity) and reinforced by radiative levitation (Charpinet et al. 1996, 1997). The $\mathrm{sdBV}_{\mathrm{s}}$ (or V1093 Her; Green et al. 2003) stars pulsate more slowly with periods of $\sim 1-2 \mathrm{~h}$, corresponding to mid-order gravity modes. The same mechanism drives these oscillations (Fontaine et al. 2003). A few stars belong to both classes and are called hybrid pulsators, showing both $p$ - and $g$-modes (e.g., Schuh et al. 2006; see also the review by Charpinet et al. 2009a).

Thus far, asteroseismic inferences could be successfully derived only from short period $p$-mode $\mathrm{B}$ subdwarf pulsators (see, e.g., Van Grootel et al. 2008a,b; Charpinet et al. 2008; Randall et al. 2009; Charpinet et al. 2009a, and reference therein). The rapid oscillations associated with relatively large amplitudes (up to 6\% in some cases) provided more favorable conditions to perform seismic studies based on data obtained from the ground. With the advent of space-borne high photometric accuracy instruments such as CoRoT (Baglin et al. 2006) and Kepler (Gilliland et al. 2010), the application of asteroseismology to the long-period sdB pulsators has become unlocked. Prior to this "space age" of sdB asteroseismology, despite heroic efforts from the ground (Randall et al. 2006a,b; Baran et al. 2009), it had indeed proved extremely difficult to differentiate the $g$-mode pulsation frequencies from the many aliases introduced by the lack of continuous coverage, particularly in view of the much longer periods and the very low amplitudes (typically $\sim 0.1 \%$ ) involved.
This difficulty was overcome with the first detailed asteroseismic solutions for long period $g$-mode sdB pulsators now becoming available, based on either Kepler data (Van Grootel et al. 2010a, for the star KIC05807616, alias KPD1943+4058) or CoRoT observations (Charpinet et al. 2010; and Van Grootel et al. 2010b, for the star KPD 0629-0016). These analyses confirm the great potential of $g$-mode asteroseismology that was envisioned for these stars. Gravity modes, because they propagate into the deep core, as opposed to $p$-modes, which remain confined to the outermost layers (Charpinet et al. 2000), have the potential to reveal the structure of the deepest regions, including the thermonuclear furnace. Van Grootel et al. (2010a,b) show that important constraints on the inner core, such as its chemical composition (related to the age of the star) and its size, are indeed accessible, suggesting in particular that the $\mathrm{He} / \mathrm{C} / \mathrm{O}$ core may be larger than expected. This would imply that efficient extra mixing processes (e.g., core convection overshoot, semi-convection) are effective.

These pioneering works constitute the very first steps in the seismic exploitation of $g$-mode $\mathrm{sdB}$ pulsators. The Kepler mission is providing the Kepler AsteroSeismic Consortium (KASC) with more than a dozen sdB stars with long period $g$-mode pulsations. The KASC working group 11 (WG11), in charge of the compact pulsators, has reported on these discoveries in several publications (Østensen et al. 2010; Kawaler et al. 2010b; Reed et al. 2010; Kawaler et al. 2010a; Østensen et al. 2011; Baran et al. 2011). In addition, a global investigation of the period spacings observed in these stars is also proposed by Reed et al. (2011). In the present paper, we focus on one of these pulsators: KIC02697388 (referred to as J190907.14+375614.2 in the SDSS catalog; Stoughton et al. 2002). This relatively faint star $\left(K_{p}=15.39\right.$ in the Kepler Input Catalog), spectroscopically identified as a rather cool B subdwarf by Østensen et al. (2010), was first observed photometrically and discovered to be pulsating during the Kepler Q2.3 exploratory run. It exhibits a remarkably rich frequency spectrum (Reed et al. 2010) that quite naturally makes it one of the most interesting objects in the Kepler sample for a detailed asteroseismic study. We present in Sect. 2, new dedicated spectroscopy of KIC02697388 and a thorough reanalysis of the Kepler Q2.3 light curve. Both constitute the basis of our detailed asteroseismic analysis of this star that is discussed in Sect. 3. We summarize our results and conclude in Sect. 4.

\section{Spectroscopic and photometric properties}

\subsection{Spectroscopy}

Independent and accurate spectroscopic measurements to estimate atmospheric parameters such as the effective temperature and the surface gravity are essential for dealing with the degeneracies generally encountered in the seismic analysis of $\mathrm{sdB}$ pulsators (see, e.g., Charpinet et al. 2005). The first determination of the surface parameters of KIC02697388 appears in Østensen et al. (2010). On the basis of a low signal-to-noise spectrum (primarily obtained for stellar classification) and using a grid of LTE model atmospheres to fit the Balmer and helium lines, these authors estimate that $T_{\mathrm{eff}}=23900 \pm 300 \mathrm{~K}$, $\log g=5.32 \pm 0.03(\mathrm{cgs})$, and $\log N(\mathrm{He}) / N(\mathrm{H})=-2.9 \pm 0.1$ for this star.

As part of a long-term program to characterize hot B subdwarfs in general, and Kepler sdB targets in particular, we obtained two $30 \mathrm{~min}$ spectra of KIC02697388 on UT 2010 June 15 and June 17, using the Steward Observatory $2.3 \mathrm{~m}$ Bok Telescope on Kitt Peak, Arizona. The combined spectrum has fairly low resolution $(R \sim 580)$, but moderately high sensitivity 
$(S / N \sim 173)$ over the total wavelength range $\sim 3600$ to $6900 \AA$ and somewhat higher sensitivity blueward of $5000 \AA(S / N \sim$ 199). We analysed the spectrum using new grids of NLTE model atmospheres and synthetic spectra developed to study hot subdwarfs of the B and $\mathrm{O}$ types. These models were constructed with the public codes TLUSTY and SYNSPEC (Hubeny \& Lanz 1995; Lanz \& Hubeny 1995). Some details are provided in Brassard et al. (2010) and Latour et al. (2010).

For sdB stars, the most accurate spectral fits that we can currently achieve are based on a grid of models that has a fixed metallicity inspired by the results of Blanchette et al. (2008). These authors used FUSE spectroscopy and suitable NLTE model atmospheres to determine the abundances of several astrophysically important elements in the atmospheres of five typical long-period pulsating sdB stars. We recall here that hot subdwarf stars are all chemically peculiar; none exhibits a solar metallicity. The five $g$-mode pulsators stars analysed by Blanchette et al. (2008) show very similar abundance patterns (see, e.g., their Fig. 6), and from their results we derived a representative composition using the most abundant heavy elements. Hence, we assumed atmospheres containing C (1/10 solar), N (solar), O (1/10 solar), Si (1/10 solar), S (solar), and Fe (solar). We are not suggesting that this composition applies in detail to KIC002697388. This metallicity should instead be seen as representative of the global effects of metals in the atmospheres of sdB stars, particularly of long-period pulsating objects such as here.

Among others, a 3D grid of 1440 NLTE model atmospheres and synthetic spectra was constructed for this specific metallicity. The grid consists of 16 grid points in $T_{\text {eff }}$ spanning a range of $20000-50000 \mathrm{~K}$ in steps of $2000 \mathrm{~K}, 10$ values of $\log g$ covering the range 4.6-6.4 in steps of $0.2 \mathrm{dex}$, and 9 values of $\log N(\mathrm{He}) / N(\mathrm{H})$ spanning the range of -4.0 to 0.0 in steps of 0.5 dex. For comparison purposes (see below), two other similar grids were also used, one with NLTE models but no metals, and the other one based on the LTE approximation and no metals. For each grid, we fitted our observed spectrum in 3D space, with the help of a $\chi^{2}$ minimization technique first developed by Pierre Bergeron (see Saffer et al. 1994, for details). The upper panel of Fig. 1 shows the best fit we obtained for KIC02697388, leading to $T_{\text {eff }}=25395 \pm 227 \mathrm{~K}, \log g=5.500 \pm 0.031(\mathrm{cgs})$, and $\log N(\mathrm{He}) / N(\mathrm{H})=-2.767 \pm 0.122$. We point out that the quoted uncertainties are formal errors in the fits and do not include systematic effects that remain difficult to evaluate. To provide a measure of the effects of metals, we also fitted our spectrum using equivalent NLTE models, but without metals (lower panel of Fig. 1). The values now come out as $T_{\text {eff }}=25505 \pm 264 \mathrm{~K}$, $\log g=5.500 \pm 0.031(\mathrm{cgs})$, and $\log N(\mathrm{He}) / N(\mathrm{H})=-2.757 \pm$ 0.122 , indicating that the presence of metals (at least with the amounts assumed) is not a critical issue in the determination of the atmospheric parameters of KIC02697388. The map shown in Fig. 1 of Brassard et al. (2010) indicates that, at the values of $T_{\text {eff }}$ and $\log g$ inferred for that star, the effects of metal blanketing are indeed quite small in $\mathrm{sdB}$ atmospheres. Likewise, we find that NLTE versus LTE models lead to similar results; in the latter case, using our grid of LTE models with no metals, we find that $T_{\text {eff }}=25688 \pm 276 \mathrm{~K}, \log g=5.517 \pm 0.031(\mathrm{cgs})$, and $\log N(\mathrm{He}) / N(\mathrm{H})=-2.719 \pm 0.118$. We do not know why our estimates of the atmospheric parameters of KIC02697388 differ significantly from those given in Østensen et al. (2010).

Figure 2 shows a sample of short period (in blue) and long period (in red) sdB pulsators whose surface parameters have been derived using the same telescope/instrument combination, reduction procedure, and grid of model atmospheres, thereby
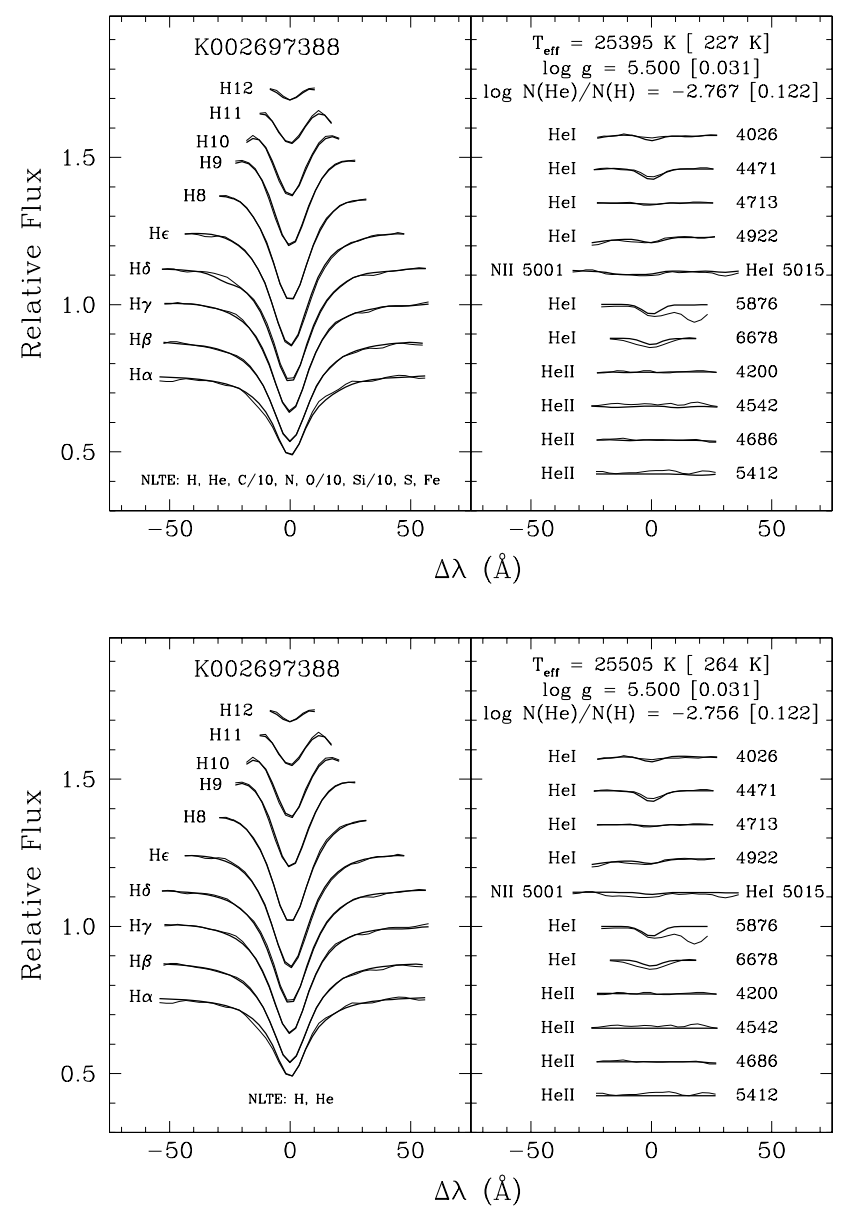

Fig. 1. Upper panel: model fit (heavy curve) to all the hydrogen and strong helium lines (thin curve) available in our high $\mathrm{S} / \mathrm{N}$, lowresolution optical spectrum of KIC 02697388. The fit was done using a 3D grid of NLTE synthetic spectra $\left(T_{\text {eff }}, \log g, \log N(\mathrm{He}) / N(\mathrm{H})\right)$ in which the abundances of $\mathrm{C}, \mathrm{N}, \mathrm{O}, \mathrm{S}, \mathrm{Si}$, and $\mathrm{Fe}$ were held fixed at amounts consistent with Blanchette et al. (2008). Lower panel: similar, but for a $3 \mathrm{D}$ grid of NLTE synthetic spectra without metals.

forming a homogeneous set. This homogeneity is a valuable property that ensures the position of each star relative to the others in the $\log g-T_{\text {eff }}$ plane should be correct, even though systematics of unknown nature may affect the determination of their parameters on an absolute scale. The atmospheric parameters derived for KIC02697388 (represented as a green square with a cross in the figure) place the star among the coolest pulsating hot B subdwarfs. Its position in the diagram is consistent with the presence of long period oscillations.

\subsection{Kepler time series photometry}

The hot B subdwarf star KIC02697388 was observed by Kepler in short cadence mode (58.8 s sampling rate) over a time baseline of $\sim 27.11$ days $(\sim 650.73 \mathrm{~h})$ from August 20 to September 16, 2009 (run Q2.3; see Østensen et al. 2010). The data were processed through the Kepler Science Processing Pipeline (Jenkins et al. 2010). A preliminary analysis of the light curve obtained for this star was presented in Reed et al. (2010) as part of a general report on the frequencies observed in several long period $\mathrm{sdB}$ pulsators discovered during the first part of the Kepler exploratory program (Q0, Q1, and Q2 runs). To exploit these data 


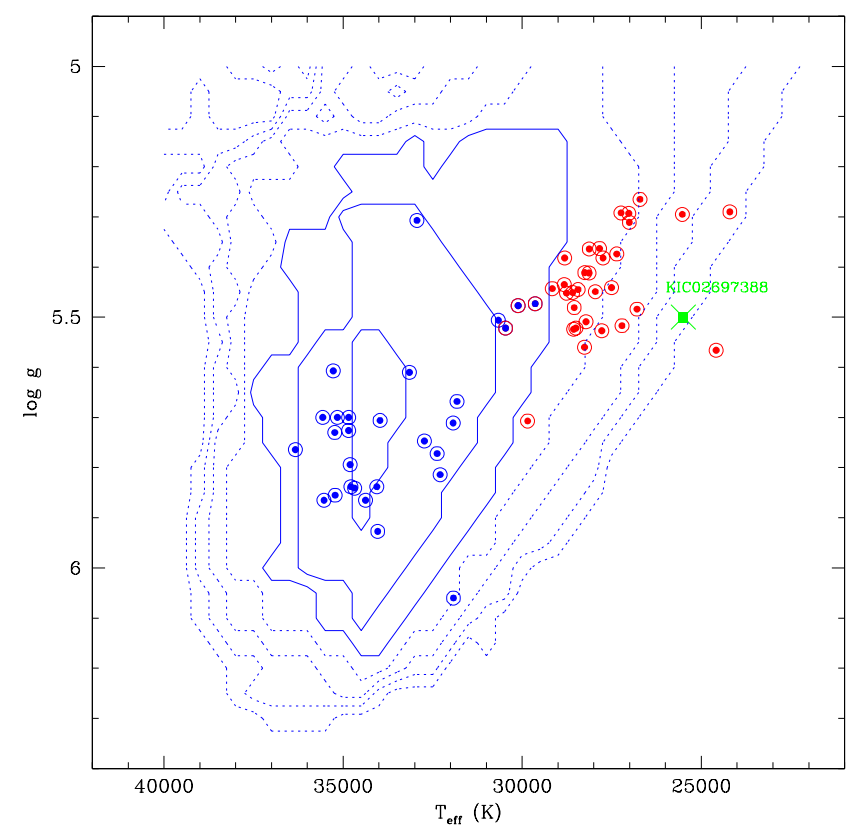

Fig. 2. Distribution of the hot B subdwarf pulsators in the $\log g-T_{\text {eff }}$ plane from a sample of 28 short-period (out of 51 known; in blue) and 33 long-period (out of 45 known; in red) sdB pulsators. Three stars in this sample show both long- and short-period pulsations (blue filled circles within a red annulus). We stress that the plotted stars form a homogeneous sample in terms of the determination of their atmospheric parameters (based on our NLTE metal-free $\mathrm{H} / \mathrm{He}$ model atmospheres). On the same homogeneous scale, the green square with a cross marks the position of KIC02697388. The overplotted blue contours indicate the number of driven $\ell=0 p$-modes (the largest contour corresponds to one driven mode) and manifest the $p$-mode instability region derived from models assuming iron distributions at equilibrium between gravitational settling and radiative levitation (Charpinet et al. 2001). Nearly all $p$-mode pulsators are concentrated within the three highest contours (solid lines) where driving is most efficient.

for a detailed asteroseismic study of KIC02697388, we need a more thorough frequency analysis that we provide here.

Figure 3 shows a 20-day-long section of the Kepler "white light" photometry of KIC02697388. We based the following analysis on the raw light curve. The choice of using raw data instead of data preliminary corrected for contamination has no impact on the seismic analysis. The only significant difference appears in the measured amplitudes of the modulations, which, for this star, are found to be larger by $\sim 20 \%$ for the corrected data. To maximize the signal-to-noise ratio of the data in Fourier space, the light curve was detrended for residual long-term variations and cleaned of data points that differ significantly from the local standard deviation by applying a running 3- $\sigma$ clipping filter. After this treatment, an effective duty cycle of $\sim 96 \%$ is reached, resulting in a window function so pure that aliasing due to interruptions never interfere with the identification of real frequencies. In this light curve, low-amplitude multi-periodic oscillations with dominant periodicities around $1-2 \mathrm{~h}$ are clearly seen. The complex interference pattern indicates that a significant number of modes are involved in the brightness modulation, as outlined by Reed et al. (2010), who conclude that at least 37 frequencies must be present.

A Lomb-Scargle periodogram (LSP; Scargle 1982) of the light curve confirms the complex multi-periodic nature of the star brightness modulation. The region where dominant signal is clearly detected covers the range $60-400 \mu \mathrm{Hz}$ (left panel of Fig. 4). The timescale of these modulations ( $\sim 1-4 \mathrm{~h})$ is typical of the variations induced by $g$-mode pulsations in long period sdB pulsators. Another frequency region also shows modulations with weak but significant signal in the $500-1100 \mu \mathrm{Hz}$ range (right panel of Fig. 4). More surprisingly, as noted by Reed et al. (2010), we also find a very weak periodic signature at a much shorter timescale (see Fig. 5), which, if real, is quite unexpected, at first sight, for a cool sdB star such as KIC02697388. This frequency would indeed be comparable to variations generally encountered in the short period $p$-mode sdB pulsators, which are found at higher effective temperatures. We provide evidence that this short period modulation is indeed very likely to be caused by an excited acoustic mode, making KIC02697388 a hybrid sdB pulsator (see Sect. 3.4). All other frequency regions, not illustrated here, up to the Nyquist limit $(\sim 8500 \mu \mathrm{Hz})$ are otherwise found to be consistent with noise.

We applied the usual prewhitening and nonlinear least squares fitting techniques to extract the frequencies (Deeming 1975). We used a dedicated software program, FELIX (Frequency Extraction for LIghtcurve eXploitation) developed by one of us (S.C.), which greatly eases and accelerates the application of this procedure, especially for long time series obtained from space (Charpinet et al. 2010). The procedure was performed with no major difficulty, thanks to the very high quality of the Kepler photometry. However, on several occasions, groups of seemingly unresolved peaks were encountered, as already mentioned in Reed et al. (2010). Dealing with close peaks that differ by less than $\sim 1.5$ times the formal resolution of the data (which is $\sim 0.43 \mu \mathrm{Hz}$ in the present case) can be problematic. In several instances, the simultaneous nonlinear least squares fitting method could not converge for the close frequencies. Our approach in these cases was to first select the dominant frequency of a crowded complex, perform the simultaneous nonlinear least squares fit on the frequency, amplitude, and phase, and freeze the frequency to the value obtained (keeping amplitude and phase as free parameters). Other close peaks of lower amplitude associated with that complex could then be selected, fitted individually for frequency, amplitude, and phase and then fitted simultaneously with the other peaks while keeping their frequency locked. This procedure allowed us to extract all the peaks in the Fourier domain beyond a given detection threshold where we used, as usual, $4 \sigma$ above the mean noise level. Nonetheless, one must keep in mind that the uncertainties associated with the frequencies derived for the unresolved peaks are, in fact, somewhat larger (around the formal resolution of the run, to be conservative) and that, except for the main peak of a complex that is certainly real, the existence of some of the lower amplitude components may be questionable. We stress however that these uncertainties only marginally affect our subsequent seismic analysis (see the next section).

Table 1 lists the 63 peaks that we extracted from the Kepler light curve. The table also provides their attributes: frequency, period, amplitude, and phase (with their error estimates $\sigma_{f}, \sigma_{P}$, $\sigma_{A}$, and $\sigma_{\mathrm{Ph}}$, respectively), as well as the signal-to-noise ratio of the detection. Among the 63 frequencies, four (labeled " $a_{n}$ ") are known as instrumental artifacts ${ }^{1}$ and 59 (labeled " $f_{n}$ ", where $n$

\footnotetext{
${ }^{1} a_{1}, a_{2}$, and $a_{4}$ are harmonics of an electronic crosstalk with the long cadence readout operation that occurs with a frequency $f_{\mathrm{LC}}=$ $566.391 \mu \mathrm{Hz} . a_{3}$ is likely another artifact of unclear origin since it is observed in the light curves of several other stars (see Østensen et al. 2010).
} 


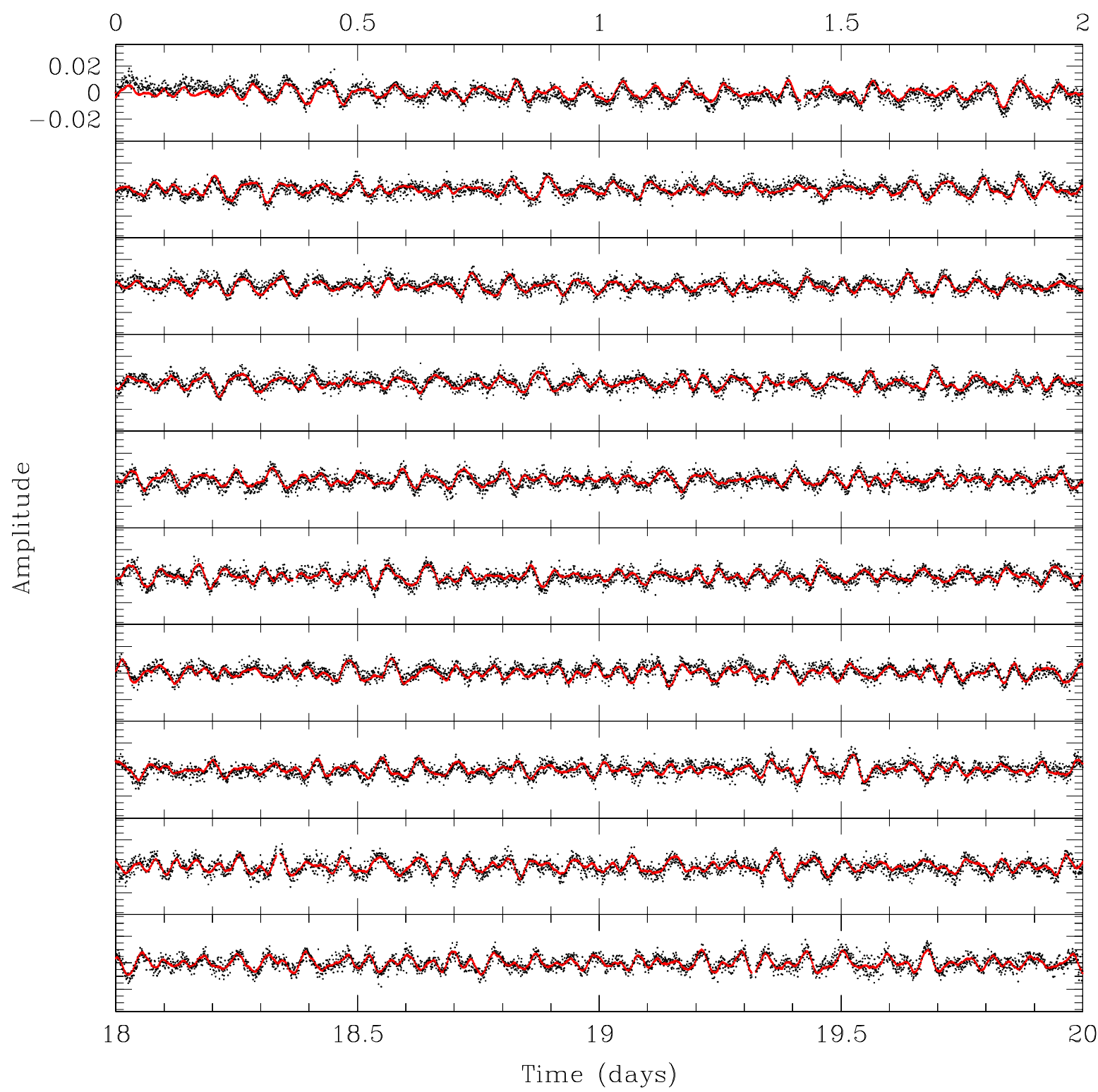

Fig. 3. Twenty day section of the light curve obtained for KIC02697388 during Q2.3 Kepler observations. The amplitude as a function of time is expressed in terms of the residual relative to the mean brightness intensity of the star. The red curve shows the reconstructed signal based on the extracted frequencies, amplitudes, and phases given in Table 1.

is ranked in order of the decreasing amplitude of the main peak) are presumably pulsation modes. In this table, close unresolved frequencies separated by less than $\sim 1.5$ times the formal resolution (i.e., $\sim 0.65 \mu \mathrm{Hz}$ ) are grouped together. We also note that the phase is relative to the beginning of the run. This zero point corresponds, in the solar system barycentric reference frame, to BJD 2455064.3628260 (time standard is UTC) and the fitted waves have the form $A \cos [2 \pi / P(t-$ phase $)]$. The noise level is extremely low, ranging from $\sim 0.0054 \%$ (54 ppm) at low frequencies to $\sim 0.0027 \%$ (27 ppm) at high frequencies. The reconstructed light curve based on all the harmonic oscillations given in Table 1 is shown in Fig. 3 plotted (in red) over the observed light curve. In a similar way, Fig. 4 shows the Lomb-Scargle periodogram of the observed time series in relevant frequency regions and, plotted upside-down, its reconstruction based on all frequencies from Table 1 . The LSP of the residual light curve after subtracting all oscillations is also given, shifted downward. In both the time domain (Fig. 3) and frequency space (Fig. 4), the reconstruction based on the fitted modulations closely reproduces the observations.

The comparison of our Table 1 with Table 3 of Reed et al. (2010) finds excellent agreement. In our present analysis, however, 22 additional frequencies (listed within brackets in Table 1) are not reported in Reed et al. (2010). The reasons are twofold: 1) we used a different estimate of the detection threshold, the one adopted by Reed et al. (2010) being slightly more conservative, and 2) in their preliminary analysis, Reed et al. (2010) did not extract all the apparent peaks in the poorly resolved groups of close frequencies.

\section{Seismic analysis}

\subsection{Method and models}

For the seismic analysis, we adopt a variant of the forwardmodelling approach applied with success to the study of several 

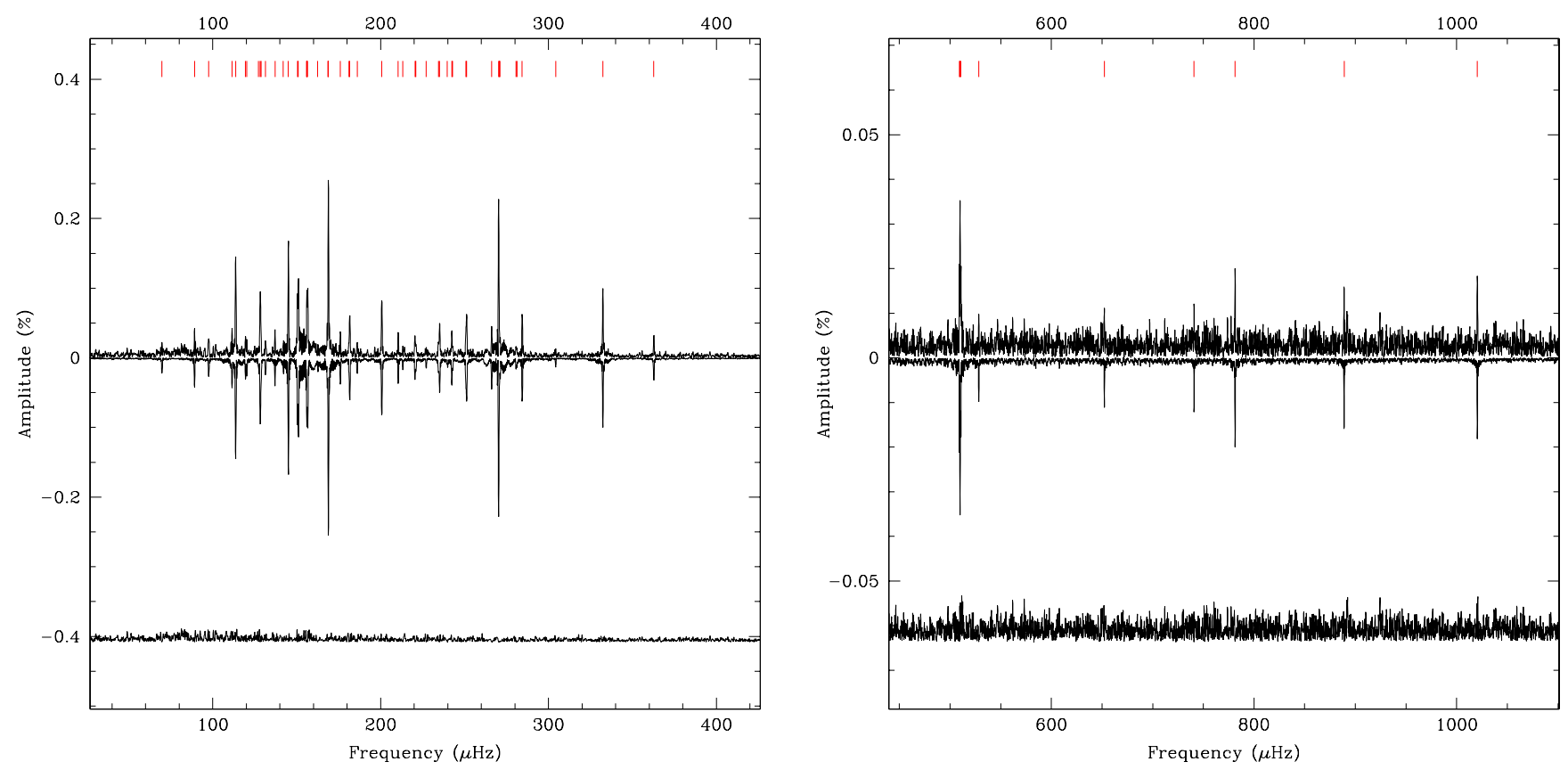

Fig. 4. Lomb-Scargle periodogram (LSP) in the 50-450 $\mu \mathrm{Hz}$ frequency range (left panel) and the 450-1100 $\mu \mathrm{Hz}$ frequency range (right panel), where signal is found. The reconstructed LSP based on the extracted harmonic oscillations given in Table 1 (indicated by red vertical segments) is shown upside down. The curve shifted downward is the LSP of the residual (i.e., noise) after subtracting all the frequencies of Table 1 from the observed light curve.

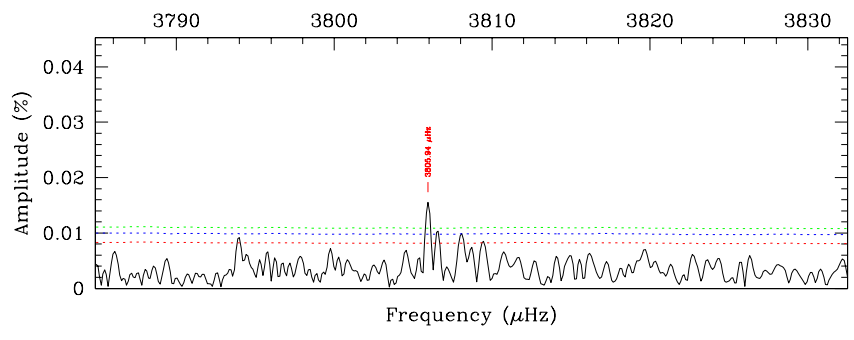

Fig. 5. Lomb-Scargle periodogram (LSP) in the $3785-3830 \mu \mathrm{Hz}$ frequency range where a weak signal is also suspected. The green (blue, red) dotted curves refer to a value equal to $4.0(3.6,3.0)$ times the local mean noise level. The indicated peak at $3805.94 \mu \mathrm{Hz}\left(f_{38}\right.$ in Table 1) clearly emerges at 5.7 times the average noise.

$p$ - and $g$-mode sdB pulsators. The method was described in detail in Charpinet et al. $(2005,2008)$ and relies on fitting simultaneously all of the observed pulsation frequencies with theoretical frequencies calculated for appropriate hot B subdwarf models. The quality of the fit is quantified using a merit function defined as

$S^{2}\left(p_{1}, \ldots, p_{n}\right)=\sum_{i=1}^{N_{\mathrm{obs}}}\left(v_{\mathrm{obs}, i}-v_{\mathrm{th}, i}\right)^{2}$,

where $N_{\text {obs }}$ is the number of observed frequencies and $\left\{v_{\mathrm{obs}, i}, v_{\mathrm{th}, i}\right\}$ are the associated pairs of observed/computed frequencies. This quantity, $S^{2}$, needs to be minimized as both a function of the frequency associations (i.e., a combinatorial first minimization required because the observed modes are not identified a priori) and as a function of the model parameters, $\left\{p_{1}, \ldots, p_{n}\right\}$. For that purpose, we developed efficient optimization codes to find the minima of the merit function (a multi-dimensional function that can be of very complex shape), which constitute the potential asteroseismic solutions. Utilizing this procedure, we obtain the mode identification (i.e., the most closely fitting association between observed and theoretical frequencies) and, more importantly, constraints on the main structural parameters of the star.

Quantitative asteroseismology of $g$-mode pulsators has become possible thanks to our so-called "third-generation" (3G) models, which is suitable for the accurate evaluation of $g$-mode pulsation frequencies. These $3 \mathrm{G}$ models are briefly described in Brassard \& Fontaine (2008, 2009). They are complete stellar structures in thermal equilibrium defined in terms of a set of parameters inspired from full evolutionary models. The reason we use static parametrized structures instead of evolutionary models is that the former provide the needed flexibility for thoroughly exploring parameter space. The input parameters needed to characterize a $3 \mathrm{G}$ model are: the total stellar mass $M_{*}$, the fractional mass of the outer hydrogen-rich envelope $\log \left(M_{\text {env }} / M_{*}\right)$, the fractional mass of the mixed convective core $\log \left(M_{\text {core }} / M_{*}\right)$, and the chemical composition of the core (with the constraint $X(\mathrm{He})+X(\mathrm{C})+X(\mathrm{O})=1)$. The effective temperature, $T_{\text {eff }}$, and surface gravity, $\log g$, are computed a posteriori for a $3 \mathrm{G}$ model of given parameters. To exploit the atmospheric parameters determined independently by spectroscopy, we incorporate the values of $T_{\text {eff }}$ and $\log g$ as external constraints in the optimization procedure to search for a best-fit model. Only the models defined by minima in the $S^{2}$ merit function that have atmospheric parameter values within a given tolerance around the spectroscopic estimates are considered acceptable, thus ensuring, by construction, consistency with spectroscopy. In the specific case of KIC02697388, acceptable solutions have to fall in the ranges of $3 \sigma$ around $T_{\text {eff }}=25395 \mathrm{~K}$ and $\log g=5.500$. We note that there is no guarantee, a priori, that a good frequency match exists within these constraints. 
Table 1. List of frequencies, $f_{n}$, and known instrumental artefacts, $a_{n}$, detected four times above the noise level.

\begin{tabular}{|c|c|c|c|c|c|c|c|c|c|}
\hline Id. & $\begin{array}{c}\text { Frequency } \\
(\mu \mathrm{Hz})\end{array}$ & $\begin{array}{c}\sigma_{f} \\
(\mu \mathrm{Hz})\end{array}$ & $\begin{array}{l}\text { Period } \\
\text { (s) }\end{array}$ & $\begin{array}{l}\sigma_{P} \\
(\mathrm{~s})\end{array}$ & $\begin{array}{c}\text { Amplitude } \\
(\%)\end{array}$ & $\begin{array}{c}\sigma_{A} \\
(\%)\end{array}$ & $\begin{array}{c}\text { Phase } \\
(\mathrm{s})\end{array}$ & $\begin{array}{c}\sigma_{\mathrm{Ph}} \\
(\mathrm{s})\end{array}$ & $S / N$ \\
\hline$\left[f_{30}\right]$ & {$[69.7703]$} & {$[0.0583]$} & [14332.754] & [11.979] & {$[0.0225]$} & {$[0.0056]$} & [5950.297] & [495.343] & {$[4.0]$} \\
\hline$f_{17}^{\dagger}$ & 89.1152 & 0.0313 & 11221.429 & 3.942 & 0.0405 & 0.0054 & 2026.985 & 216.406 & 7.5 \\
\hline$f_{26}^{\dagger}$ & 97.5648 & 0.0440 & 10249.596 & 4.624 & 0.0276 & 0.0052 & 7440.517 & 288.760 & 5.3 \\
\hline$f_{16}^{\dagger 0}$ & 111.4815 & 0.0269 & 8970.096 & 2.164 & 0.0444 & 0.0051 & 1421.345 & 158.404 & 8.8 \\
\hline$f_{4}^{\dagger}$ & 113.6474 & 0.0080 & 8799.142 & 0.616 & 0.1489 & 0.0050 & 4504.076 & 46.329 & 29.6 \\
\hline & 119.5463 & 0.0403 & 8364.957 & 2.822 & 0.0291 & 0.0050 & 2198.195 & 230.029 & 5.8 \\
\hline & 120.2611 & 0.0446 & 8315.238 & 3.084 & 0.0262 & 0.0050 & 3800.198 & 128.502 & 5.3 \\
\hline$\left[f_{29}\right]$ & [127.2358] & [0.0512] & [7859.421] & [3.160] & {$[0.0226]$} & [0.0049] & [3977.980] & [138.073] & [4.6] \\
\hline $\begin{array}{l}f_{7}^{\dagger} \\
{\left[f_{7}^{+}\right]}\end{array}$ & $\begin{array}{c}128.2844 \\
{[128.8021]}\end{array}$ & $\begin{array}{c}0.0108 \\
{[0.0233]}\end{array}$ & $\begin{array}{c}7795.183 \\
{[7763.850]}\end{array}$ & $\begin{array}{c}0.659 \\
{[1.406]}\end{array}$ & $\begin{array}{c}0.1057 \\
{[0.0491]}\end{array}$ & $\begin{array}{c}0.0049 \\
{[0.0049]}\end{array}$ & $\begin{array}{c}1595.009 \\
{[6758.897]}\end{array}$ & $\begin{array}{c}60.997 \\
{[64.285]}\end{array}$ & $\begin{array}{c}21.7 \\
{[10.1]}\end{array}$ \\
\hline$f_{25}^{\dagger}$ & 131.4261 & 0.0414 & 7608.840 & 2.395 & 0.0278 & 0.0049 & 5923.282 & 216.677 & 5.7 \\
\hline$f_{21}^{\dagger}$ & 137.0729 & 0.0332 & 7295.387 & 1.769 & 0.0340 & 0.0048 & 1127.585 & 168.294 & 7.1 \\
\hline$f_{28}^{\dagger}$ & 141.9936 & 0.0491 & 7042.572 & 2.435 & 0.0227 & 0.0047 & 707.760 & 244.406 & 4.8 \\
\hline$f_{3}^{\dagger}$ & 145.0232 & 0.0067 & 6895.449 & 0.319 & 0.1652 & 0.0047 & 3285.761 & 32.881 & 35.1 \\
\hline$f_{6}^{-}$ & 150.5912 & 0.0104 & 6640.494 & 0.458 & 0.1068 & 0.0047 & 3674.261 & 32.440 & 22.6 \\
\hline$f_{6}^{\dagger}$ & 150.9287 & 0.0097 & 6625.647 & 0.424 & 0.1149 & 0.0047 & 3737.964 & 63.872 & 24.4 \\
\hline$\left[f_{5}^{-}\right]$ & [155.8085] & {$[0.0382]$} & [6418.137] & [1.572] & [0.0286] & {$[0.0046]$} & [2184.460] & [115.094] & [6.2] \\
\hline$f_{5}^{\dagger}$ & 156.1630 & 0.0076 & 6403.564 & 0.310 & 0.1440 & 0.0046 & 1357.057 & 33.610 & 31.2 \\
\hline$f_{5}^{+}$ & 156.4483 & 0.0082 & 6391.887 & 0.336 & 0.1324 & 0.0046 & 5988.044 & 79.239 & 28.6 \\
\hline$\left[f_{31}\right]$ & [162.4817] & {$[0.0517]$} & [6154.538] & {$[1.960]$} & [0.0205] & {$[0.00$} & [3450.601] & [235.704] & [4.5] \\
\hline$\left[f_{1}^{-}\right]$ & [168.5419] & {$[0.0318]$} & [5933.243] & [1.121] & {$[0.0320]$} & {$[0.0043]$} & {$[629.466]$} & [114.867] & [7.4] \\
\hline$f_{1}^{\dagger}$ & 168.8681 & 0.0040 & 5921.782 & 0.141 & 0.2539 & 0.0043 & 1729.375 & 26.618 & 58.7 \\
\hline $\begin{array}{l}1 \\
f_{20}^{\dagger} \\
{\left[f_{12}^{-}\right]}\end{array}$ & $\begin{array}{c}176.0601 \\
{[181.2262]}\end{array}$ & $\begin{array}{c}0.0295 \\
{[0.0447]}\end{array}$ & $\begin{array}{c}5679.880 \\
{[5517.967]}\end{array}$ & $\begin{array}{c}0.953 \\
{[1.360]}\end{array}$ & $\begin{array}{c}0.0342 \\
{[0.0220]}\end{array}$ & $\begin{array}{c}0.0043 \\
{[0.0042]}\end{array}$ & $\begin{array}{c}4253.673 \\
{[5195.421]}\end{array}$ & $\begin{array}{c}130.206 \\
{[131.025]}\end{array}$ & $\begin{array}{c}8.0 \\
{[5.3]}\end{array}$ \\
\hline$f_{12}^{\dagger}$ & 181.6142 & 0.0159 & 5506.176 & 0.482 & 0.0616 & 0.0042 & 3470.998 & 87.360 & 14.8 \\
\hline$f_{27}^{\dagger}$ & 186.0600 & 0.0401 & 5374.609 & 1.158 & 0.0242 & 0.0041 & 1483.188 & 174.040 & 5.9 \\
\hline$f_{9}^{\dagger}$ & 200.6738 & 0.0108 & 4983.212 & 0.268 & 0.0845 & 0.0039 & 1001.055 & 46.077 & 21.8 \\
\hline$f_{18}^{\dagger}$ & 210.4893 & 0.0236 & 4750.836 & 0.532 & 0.0368 & 0.0037 & 1601.505 & 100.915 & 10.0 \\
\hline$\left[f_{34}\right]$ & [213.1780] & [0.0508] & [4690.916] & [1.119] & {$[0.0170]$} & [0.0037] & [1166.422] & [216.444] & [4.6] \\
\hline$f_{23}^{\dagger}$ & 220.4571 & 0.0256 & 4536.031 & 0.526 & 0.0333 & 0.0036 & 2964.239 & 112.153 & 9.2 \\
\hline$\left[\tilde{f}_{23}^{+}\right]$ & [220.9646] & [0.0430] & [4525.611] & [0.882] & [0.0197] & [0.0036] & [2085.505] & [96.636] & [5.5] \\
\hline$\left[f_{36}\right]$ & [227.1500] & {$[0.0515]$} & [4402.378] & .997] & $.0163]$ & {$[0.0036]$} & [3029.305] & [211.298] & [4.6] \\
\hline$\left[f_{14}^{-}\right]$ & [234.5017] & {$[0.0306]$} & [4264.362] & {$[0.556]$} & {$[0.0273]$} & {$[0.0036]$} & [3871.285] & {$[62.888]$} & [7.7] \\
\hline$f_{14}^{\dagger}$ & 235.1137 & 0.0154 & 4253.262 & 0.278 & 0.0545 & 0.0036 & 3040.633 & 61.923 & 15.3 \\
\hline$\left[f_{35}^{\dagger}\right]$ & [239.7070] & [0.0491] & [4171.759] & [0.855] & [0.0167] & [0.0035] & [2406.738] & [200.351] & [4.8] \\
\hline$f_{15}^{\dagger}$ & 242.4485 & & 4124.58 & & & 0.00 & 1345.5 & & 13.9 \\
\hline$\left[f_{15}^{+}\right]$ & [242.7739] & 31] & & & & {$[0.0$} & & & [7.1] \\
\hline$\left[f_{10}^{-}\right]$ & [250.8068] & {$[0.0181]$} & [3987.133] & [0.288] & [0.0443] & {$[0.0034]$} & [1160.721] & [39.687] & [13.0] \\
\hline$f_{10}^{\dagger}$ & 251.2739 & 0.0125 & 3979.721 & 0.198 & 0.0642 & 0.0034 & 136.445 & 53.243 & 18.9 \\
\hline$f_{13}^{\dagger}$ & 266.1637 & 0.0138 & 3757.087 & 0.194 & 0.0553 & 0.0032 & 3249.773 & 53.389 & 17.1 \\
\hline$f_{2}^{\dagger}$ & 270.2375 & 0.0033 & 3700.449 & 0.045 & 0.2322 & 0.0032 & 1860.908 & 17.236 & 71.9 \\
\hline$\left[f_{2}^{+}\right]$ & [270.6106] & [0.0114] & [3695.346] & [0.156] & {$[0.0666]$} & {$[0.0032]$} & & [30.939] & [20.6] \\
\hline$\left[f_{2}^{++}\right]$ & [271.0503] & [0.0297] & [3689.352] & $.404]$ & {$[0.0256]$} & [0.0032] & [838.978] & {$[60.635]$} & [7.9] \\
\hline$\left[f_{39}^{-}\right]$ & [280.5864] & {$[0.0572]$} & [3563.964] & {$[0.727]$} & [0.0129] & {$[0.0031]$} & [3118.843] & [218.949] & [4.1] \\
\hline$\left[f_{39}\right]$ & [281.1774] & {$[0.0553]$} & [3556.474] & {$[0.700]$} & [0.0134] & {$[0.0031]$} & [2659.733] & [107.796] & [4.3] \\
\hline$f_{11}^{\dagger}$ & 284.3102 & 0.0117 & 3517.285 & & 0.0622 & 0.0031 & 870.543 & 44.586 & 20.2 \\
\hline$\left[f_{40}\right]$ & [304.3486] & {$[0.0559]$} & [3285.705] & [0.603] & [0.0128] & {$[0.0030]$} & [2655.7 & [200.066] & [4.2] \\
\hline$f_{8}^{\dagger}$ & 332.3660 & 0.0068 & 3008.731 & 0.061 & 0.1023 & 0.0029 & 2894.451 & 22.928 & 34.8 \\
\hline$f_{22}^{\dagger}$ & 362.7018 & 0.0201 & 2757.086 & 0.153 & 0.0337 & 0.0029 & 56.124 & 63.959 & 11.7 \\
\hline$f_{19}^{-}$ & 509.1834 & 0.0416 & 1963.929 & 0.160 & 0.0155 & 0.0027 & 5.821 & 50.608 & 5.7 \\
\hline$f_{19}^{\dagger}$ & 509.9346 & 0.0175 & 1961.036 & 0.067 & 0.0367 & 0.0027 & 849.272 & 43.374 & 13.4 \\
\hline$f_{19}^{+}$ & 510.6214 & 0.0305 & 1958.398 & 0.117 & 0.0211 & 0.0027 & 494.302 & 37.189 & 7.7 \\
\hline$\left[f_{43}\right]$ & [528.4049] & [0.0609] & [1892.488] & [0.218] & [0.0106] & [0.0027] & [186.498] & [140.006] & [3.9] \\
\hline$\left[f_{42}\right]$ & [652.3091] & {$[0.0606]$} & [1533.016] & {$[0.142]$} & {$[0.0107]$} & {$[0.0028]$} & [1101.340] & [111.483] & [3.9] \\
\hline$f_{41}^{\dagger}$ & 740.8690 & 0.0510 & 1349.766 & 0.093 & 0.0127 & 0.0028 & 314.210 & 82.782 & 4.6 \\
\hline$f_{32}^{\dagger+1}$ & 781.3763 & 0.0323 & 1279.793 & 0.053 & 0.0203 & 0.0028 & 1048.804 & 49.103 & 7.3 \\
\hline$f_{37}^{\dagger}$ & 888.9323 & 0.0412 & 1124.945 & 0.052 & 0.0160 & 0.0028 & 831.746 & 55.031 & 5.7 \\
\hline & [1020.4164] & {$[0.0348]$} & [979.992] & [0.033] & [0.0186] & {$[0.0028]$} & [27.603] & [41.088] & [6.8] \\
\hline$f_{38}^{*}$ & 3805.9436 & 0.0410 & 262.747 & 0.003 & 0.0156 & 0.0027 & 241.101 & 13.184 & 5.7 \\
\hline
\end{tabular}


Table 1. continued.

\begin{tabular}{lccccccccc}
\hline \hline Id. & $\begin{array}{c}\text { Frequency } \\
(\mu \mathrm{Hz})\end{array}$ & $\begin{array}{c}\sigma_{f} \\
(\mu \mathrm{Hz})\end{array}$ & $\begin{array}{c}\text { Period } \\
(\mathrm{s})\end{array}$ & $\begin{array}{c}\sigma_{P} \\
(\mathrm{~s})\end{array}$ & $\begin{array}{c}\text { Amplitude } \\
(\%)\end{array}$ & $\begin{array}{c}\sigma_{A} \\
(\%)\end{array}$ & $\begin{array}{c}\text { Phase } \\
(\mathrm{s})\end{array}$ & $\begin{array}{c}\sigma_{\mathrm{Ph}} \\
(\mathrm{s})\end{array}$ & $S / N$ \\
\hline \multicolumn{2}{l}{ Instrumental artefacts } & & & & & & & & \\
$a_{2}: 8 f_{\text {lc }}$ & 4531.5510 & 0.0312 & 220.675 & 0.002 & 0.0192 & 0.0025 & 169.943 & 8.978 & 7.5 \\
$a_{1}: 9 f_{\text {lc }}$ & 5097.9751 & 0.0283 & 196.156 & 0.001 & 0.0227 & 0.0027 & 36.818 & 6.745 & 8.3 \\
$a_{3}$ & 7865.6389 & 0.0332 & 127.135 & 0.001 & 0.0186 & 0.0026 & 31.948 & 5.349 & 7.1 \\
$a_{4}: 15 f_{\text {lc }}$ & 8496.7276 & 0.0471 & 117.692 & 0.001 & 0.0138 & 0.0028 & 87.565 & 6.862 & 5.0 \\
\hline
\end{tabular}

Notes. Frequency not reported in Reed et al. (2010); ${ }^{\dagger}$ frequency selected for the first step in the search of an asteroseismic solution; ${ }^{*}$ suspected $p$-mode.

\subsection{Search for an optimal model}

In the present seismic analysis of KIC 02697388 , we adopt the following approach: first, to be conservative, we only consider a subset of frequencies (those marked with a "†" sign) that have been reported both in Table 1 and in Table 3 of Reed et al. (2010). In this way, the additional frequencies that we report do not interfere with the search of an optimal model, should some of these frequencies be spurious. A comparison can however be done afterward with the theoretical frequency spectrum of the selected solution. In a second step, we use all the independent frequencies (all the " $f_{n}$ " listed in Table 1) in the analysis. For each group of close frequencies, we retain only the dominant component (in amplitude) as an independent pulsation mode. Other close frequencies are just ignored. The unresolved frequencies can be interpreted in various ways. A possibility is that the star is rotating slowly and the non-radial pulsation modes are split because of this rotation. Another option is that the amplitude and/or phase of some modes are not constant during the run and cannot be correctly prewhitened with waves that are assumed to be purely sinusoidal. We will be unable to decide whether this is possible until longer time series on this star (which Kepler will eventually provide) become available in order to resolve properly these fine structures in the amplitude spectrum. We emphasize, however, that the uncertainties associated with these unresolved features do not have a strong impact on the seismic analysis presented here.

For the first conservative approach, this leaves us with 32 frequencies that are assumed independent, while we end up with 43 independent frequencies when considering the entire spectrum listed in Table 1. In both cases, we attempt to match these frequencies simultaneously to modes computed from perfectly spherical (i.e, nonrotating) models. With this hypothesis, all frequencies are considered as $m=0$ modes and the theoretical frequency spectrum is defined only in terms of the $k$ (radial order) and $\ell$ (degree) of the modes. We point out that, for slow rotators, the eventual error in misidentifying the $m$ index of an observed frequency has a limited impact on the results of a seismic analysis. This is true as long as the error induced on the frequency remains smaller than the overall accuracy achieved for the seismic fit, which will be the case for KIC02697388.

The search for best-fit solutions was carried out in the following domain: $M_{*} / M_{\odot} \in[0.30,0.70], \log q(H)=$ $\log \left(M_{\text {env }} / M_{*}\right) \in[-5.0,-1.8], \log q_{\text {core }}=\log \left(1-M_{\text {core }} / M_{*}\right) \in$ $[-0.40,-0.10]$, and $X_{\text {core }}(\mathrm{C}+\mathrm{O}) \in[0.00,0.99]$, where $X_{\text {core }}(\mathrm{C}+\mathrm{O})$ is the fractional part (in mass) of carbon and oxygen in the core. The ranges considered for $\log q(H)$ and $M_{*}$ rely on expectations from various formation scenarios for hot subdwarfs (Han et al. 2002, 2003), whereas the limits on the core size are loosely inspired by horizontal branch stellar evolutionary calculations (Dorman et al. 1993).
To restrict the search domain, we have to make additional assumptions about the nature of the modes that have been detected. Since the star was monitored photometrically, we usually consider from the expected visibility of the pulsation modes that only low degree modes can be effectively seen. We typically limit the search to modes of degree $\ell \leq 2$, unless we are forced to consider higher $\ell$-values. Our first calculations assuming that the modes were only $\ell=1$ and 2 led us to realize that the light curve of KIC02697388 cannot be understood in terms of $\ell \leq 2$ modes only. Within this strict limitation on $\ell$, we were unable to find a suitable simultaneous fit to the observed frequencies and we concluded that some of the modes should be of higher $\ell$. In terms of visibility, beyond $\ell=1$ and 2 , we expect to see preferentially the $\ell=4$ modes that, in sdB stars, are significantly less affected than $\ell=3$ modes by the geometric cancellation effect (Randall et al. 2005).

A closer look at the structure of the pulsation spectrum illustrated in Fig. 4 (see also Table 1) can also be instructive. In terms of amplitude distribution, we note that most of the higher amplitude frequencies are concentrated in the $100-200 \mu \mathrm{Hz}$ range. Between 200 and $380 \mu \mathrm{Hz}$, the amplitudes of the modes generally appear to be smaller, except for one frequency, $f_{2}$ at $270.24 \mu \mathrm{Hz}$, which is the second highest peak. The range 380$500 \mu \mathrm{Hz}$ forms a gap where no pulsation is detected and, again, peaks are found in the $500-1050 \mu \mathrm{Hz}$ domain, but all of them have an extremely small amplitude (the amplitude scale in the right panel of Fig. 4 is considerably wider than the left panel). Interestingly, this observed amplitude distribution can be linked with some theoretical expectations. Fontaine et al. (2003) provided an extensive study of the driving mechanism responsible for the $g$-mode instabilities in hot B subdwarfs. Apart from the well-known discrepancy between the locations of the observed and theoretical instability strips that can possibly be resolved with the inclusion of nickel, in addition to iron, as a significant source of opacity (Jeffery \& Saio 2006a,b, 2007; Charpinet et al. 2009a), Fontaine et al. (2003) show that the range of frequency (period) where the $g$-modes are driven depends on the degree $\ell$. In particular, Fig. 9 of Fontaine et al. (2003) clearly indicates that as $\ell$ increases, shorter periods (higher frequencies) are driven. This behavior occurs because the excitation mechanism acting on these $g$-modes drives a range of radial orders rather than a range of periods (frequencies) and, in the asymptotic regime, the periods of modes with the same $k$ value scale approximately as $[\ell(\ell+1)]^{-0.5}$. It is therefore tempting to interpret the observed frequency spectrum of KIC02697388 as the superposition of 3 series of modes of roughly the same range of radial order $k$ but with $\ell=1,2$, and 4 . Since each series would be shifted by a factor $[\ell(\ell+1)]^{0.5}$ from lower $(\ell=1)$ to higher frequencies $(\ell=4)$, with possibly a gap for the missing (i.e., hardly detectable) $\ell=3$ modes, this scheme matches quite well, at least qualitatively, the observed structure of the pulsation 
S. Charpinet et al.: Deep asteroseismic sounding of the sdB pulsator KIC02697388 from Kepler time series photometry
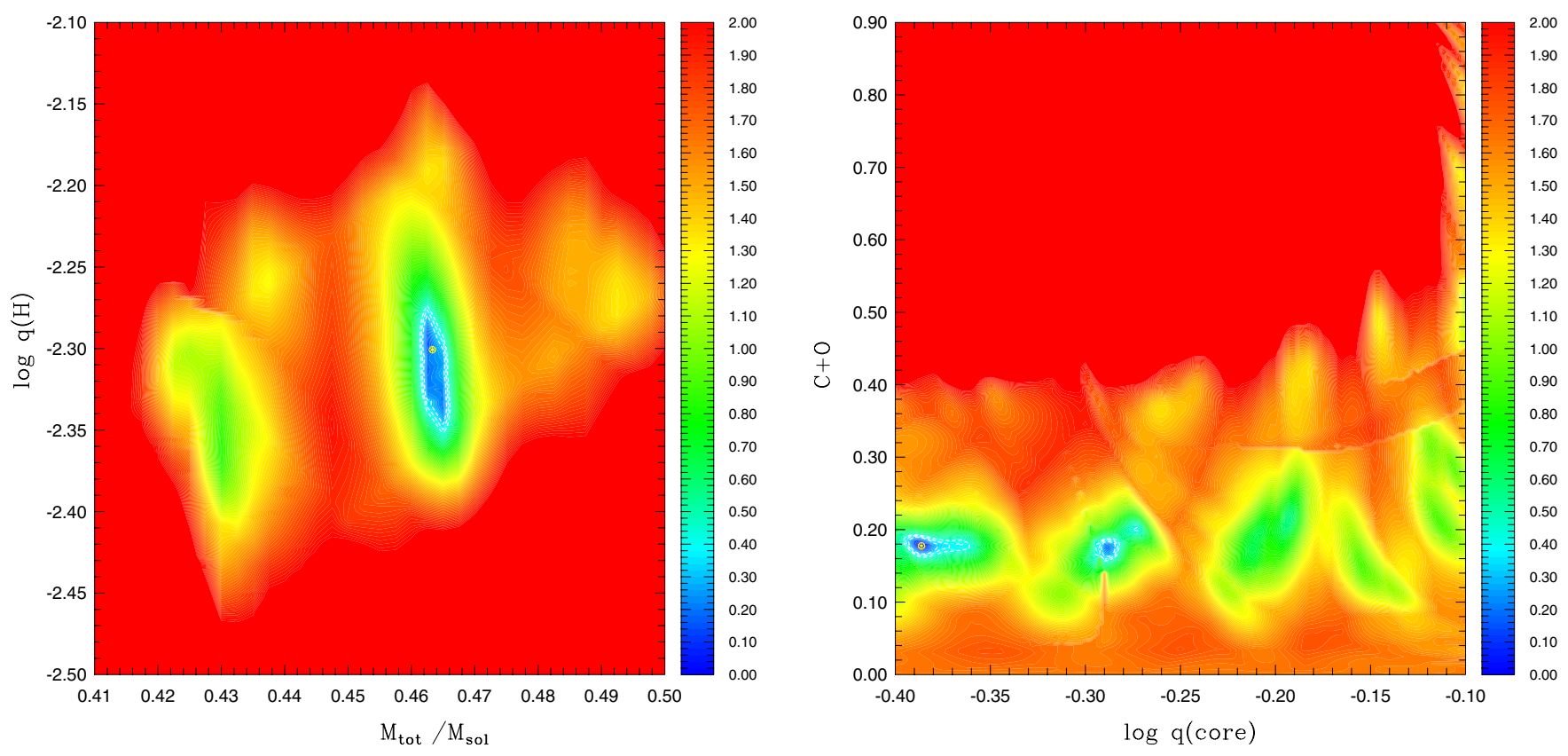

Fig. 6. Left panel: slice of the $S^{2}$ function (in logarithmic units) along the $M_{*}-\log q(H)$ plane with the parameters $\log q(\operatorname{core})$ and $X_{\text {core }}(\mathrm{C}+\mathrm{O})$ fixed to their optimal values obtained in the best-fit seismic model 1. Right panel: slice of the $S^{2}$ function $($ in $\log )$ along the $\log q(\operatorname{core})-X_{\text {core }}(\mathrm{C}+\mathrm{O})$ plane with the parameters $M_{*}$ and $\log q(H)$ fixed to their optimal values. White contours show regions where the frequency fits have $S^{2}$ values within, respectively, the $1 \sigma, 2 \sigma$, and $3 \sigma$ confidence levels relative to the best-fit solution.

spectrum. Keeping these considerations in mind, we therefore consider for our forward modelling exploration that frequencies lower than $120 \mu \mathrm{Hz}$ should be $\ell=1$ modes, those lower than $500 \mu \mathrm{Hz}$ should be either $\ell=1$ or $\ell=2$ pulsation modes, and above $500 \mu \mathrm{Hz}$, we allowed the frequencies to be associated with modes of degree $\ell=1,2$, or 4 . Associations with $\ell=4$ modes were also permitted for five frequencies (namely, $f_{40}, f_{39}, f_{35}$, $f_{36}$, and $f_{34}$ ) because of their very low apparent amplitudes. In summary, this approach allows the very low amplitude frequencies to be $\ell=4$ modes, while there is still a possibility that they are associated with modes of degree $\ell=1$ or 2 , if these mode frequencies fit better.

Within the search domain specified, and taking into consideration the external constraints on atmospheric parameters, we first ran the optimization code on the reduced set of 32 frequencies (i.e., the conservative approach). This search revealed that two families of models provide the most accurate possible match to the considered frequencies. The main parameters of these two model solutions are summarized in Table 2. Both solutions turn out to be essentially equal in terms of quality of fit (the value of $S^{2} / N_{\text {obs }}$ ). The frequency match (not given here) shows that the two families differ slightly at the level of the mode identification, but do not provide additional arguments that would allow us to favor one of the solutions over the other. Looking at the 11 additional frequencies that were not considered in this first exploration, we realized that all of them can be associated with modes present in the theoretical pulsation spectrum of both families of models, without their quality of fit being degraded significantly. This a posteriori match is highly unlikely to occur by chance and we interpret it as a clear indication that these additional frequencies not reported in Reed et al. (2010) are real. In this context, we re-ran the optimization code using the full set of 43 frequencies (i.e., including all the independent frequencies identified in our analysis presented in Sect. 2). This new search, as expected, also uncovered evidence of two families of solutions with only slightly differing parameters. These solutions are
Table 2. The two families of solutions based on 32 modes or 43 modes.

\begin{tabular}{lccccc}
\hline \hline & \multicolumn{2}{c}{ Model 1 } & & \multicolumn{2}{c}{ Model 2 } \\
& 32 modes & 43 modes & & 32 modes & 43 modes \\
\cline { 2 - 3 } \cline { 5 - 6 }$M_{*}\left(M_{\odot}\right)$ & 0.4643 & 0.4633 & & 0.4550 & 0.4520 \\
$\log q(H)$ & -2.295 & -2.301 & & -2.347 & -2.350 \\
$\log q_{\text {core }}$ & -0.385 & -0.386 & & -0.291 & -0.301 \\
$X(\mathrm{C}+\mathrm{O})$ & 0.168 & 0.178 & & 0.261 & 0.268 \\
& & & & & \\
$T_{\text {eff }}(\mathrm{K})$ & 25622 & 25622 & & 25616 & 25555 \\
$\log g$ & 5.488 & 5.488 & & 5.495 & 5.499 \\
$S^{2} / N_{\text {obs }}$ & 0.93 & 0.99 & & 1.02 & 1.25 \\
\hline
\end{tabular}

also given in Table 2. The models obtained by simultaneously fitting 32 modes or 43 modes clearly do not differ significantly. With 43 modes, model 1 may appear slightly better than model 2 in terms of quality of fit $\left(S^{2} / N_{\text {obs }}\right.$ value), but the difference is insignificant and we objectively cannot, at this stage, select one of these models as the true solution. We emphasize that both solutions show excellent agreement with spectroscopic estimates of the effective temperature and surface gravity.

The maps shown in Figs. 6 and 7 illustrate the behavior of the merit function in the vicinity of each best-fit seismic solution. In both panels, the merit function $S^{2}$ incorporates the spectroscopic constraints on atmospheric parameters. To create these plots, we tolerated a deviation of $3 \sigma$ for the effective temperature and $2 \sigma$ for the surface gravity. An exponential correction factor multiplies the merit function if the model effective temperature and surface gravity are outside these ranges, in effect degrading the $S^{2}$ value of the model. The panels clearly indicate deep blue regions (corresponding to best-fitting models, i.e., low values of $S^{2}$ ) in the $M_{*}-\log q(H)$ and $\log q($ core $)-X_{\text {core }}(\mathrm{C}+\mathrm{O})$ planes, which are well-defined by the pulsation spectrum and the spectroscopic constraints. The regions in red correspond to models that are inconsistent with spectroscopic values within the tolerance mentioned above or that provide a very poor match to the 


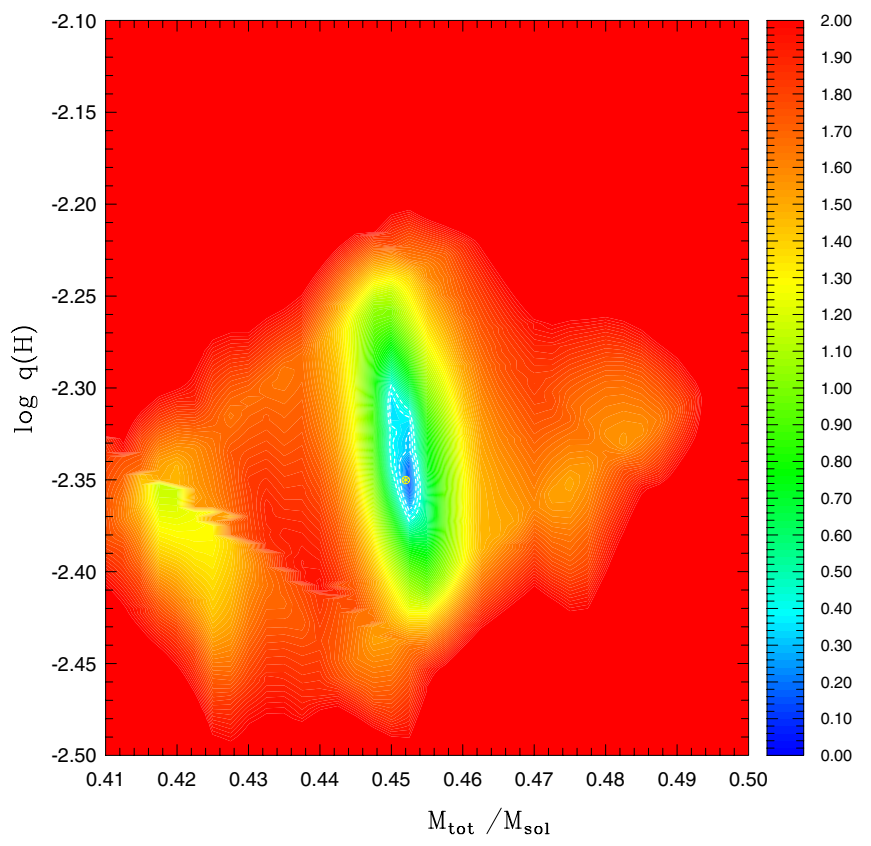

Fig. 7. Same as Fig. 6 but for model solution 2.

observed frequencies. The two uncovered valleys clearly show solutions that are significantly better in reproducing the observed pulsation spectrum than any other surrounding area of the parameter space. To verify whether the two solutions are truly distinct, we calculated a large 4-dimensional grid of 333268 models focusing on the region, in parameter space, that contains the two identified models. Figure 8 shows a map representing the "projection" $\left(\underline{S}^{2}\right.$, in logarithmic scale) of the 4D $S^{2}$ function constructed from this grid on the $\log q($ core $)-X_{\text {core }}(\mathrm{C}+\mathrm{O})$ plane. This projection is defined as

$\underline{S}^{2}\left(p_{1}, p_{2}\right)=\min \left\{S^{2}\left(p_{1}, p_{2}, p_{2}, p_{3}\right) ; \forall p_{2}, \forall p_{3}\right\}$,

where $p_{1}=\log q($ core $), p_{2}=X_{\text {core }}(\mathrm{C}+\mathrm{O}), p_{3}=M_{*}$, and $p_{4}=\log q(H)$. In other words, since we are interested in the minima of the merit function (the best-fit models), at each locus of the represented plane, the value given $\left(\underline{S}^{2}\right)$ is the lowest value of $S^{2}$ among those found for all $M_{*}$ and $\log q(H)$ varied independently. This map illustrates that the two families of solutions are indeed unconnected valleys. The two regions of best-fit models (dark blue areas) remain confined to quite narrow ranges for the $\log q$ (core) parameter, but show some elongation in the $X_{\text {core }}(\mathrm{C}+\mathrm{O})$ direction. The domain confined within the $1 \sigma$ contours provides, for each solution, a conservative estimate of the internal precision at which these parameters are effectively measured for KIC02697388.

Similarly, Fig. 9 shows the "projection" of $S^{2}$, but this time onto the $M_{*}-\log q(H)$ plane. The represented quantity is now

$\underline{\mathrm{S}}^{2}\left(p_{2}, p_{3}\right)=\min \left\{S^{2}\left(p_{1}, p_{2}, p_{2}, p_{3}\right) ; \forall p_{1}, \forall p_{2}\right\}$,

where the $\left\{p_{j}\right\}$ 's are defined as before, i.e., at each locus of the represented plane, the value given $\left(\underline{S}^{2}\right)$ is the lowest value of $S^{2}$ among those found for all $\log q($ core $)$ and $X_{\text {core }}(\mathrm{C}+\mathrm{O})$ varied independently. The two solutions clearly overlap in the $M_{*}-\log q(H)$ plane, forming a joint area. The domain confined within the $1 \sigma$ contours provides a conservative estimate of the internal precision at which $M_{*}$ and $\log q(H)$ are effectively measured for KIC02697388 (see Sect. 3.5).
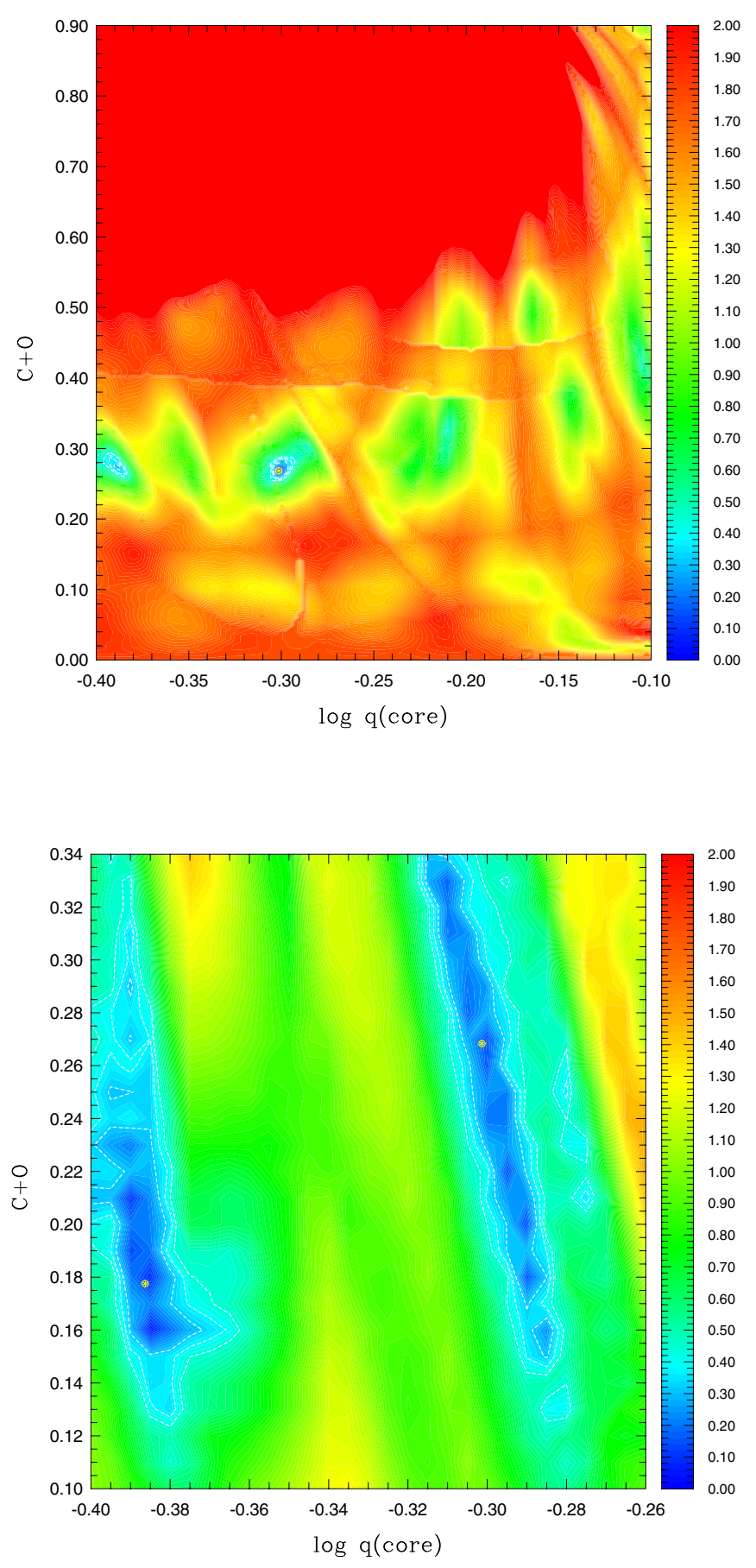

Fig. 8. Expanded view of the $\log q($ core $)-X_{\text {core }}(\mathrm{C}+\mathrm{O})$ region where the two model solutions are found. The map represents $\underline{S}^{2}$, a "projection" of the 4-dimensional $S^{2}$ function (in $\log$ scale): at each $\log q$ (core), $X_{\text {core }}(\mathrm{C}+\mathrm{O})$ position, the value given is the minimum of $\log S^{2}$ that can be found among the values obtained for all $M_{*}$ and $\log q(H)$. White contours show regions where the frequency matches have $S^{2}$ values within, respectively, the $1 \sigma, 2 \sigma$, and $3 \sigma$ confidence levels relative to the bestfit solution. The yellow marks indicate the positions of the two solutions found by the optimization code.

\subsection{Frequency match and mode identification}

The two model solutions isolated for KIC02697388 provide very good simultaneous matches to the 43 observed frequencies (but see below). Details of both the fit and mode identification are 


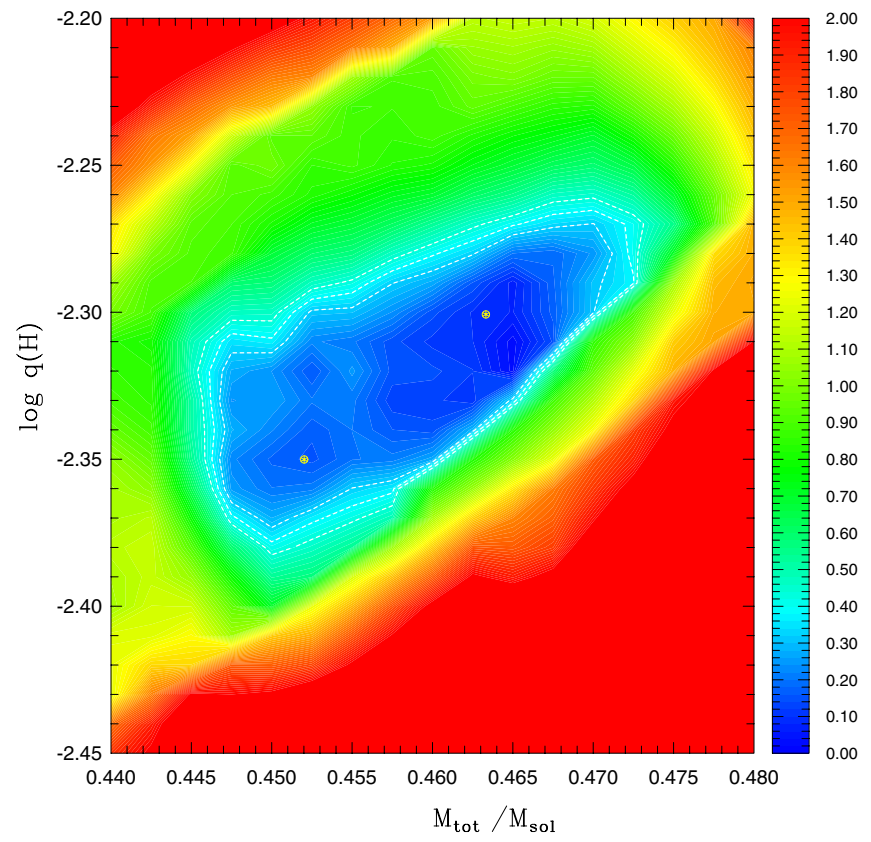

Fig. 9. Same as Fig. 8 but the projection is done onto the $M_{*}-\log q(H)$ plane.

given, for both models, in Tables 3 and 4, respectively (these tables are provided as online material only). Figure 10 also provides a graphical illustration of the fits. These tables list the most relevant computed frequencies $v_{\text {th }}$ (periods; $P_{\text {th }}$ ) with some useful properties (such as the kinetic energy, $\log E_{\text {kin }}$, and the Ledoux coefficient, $C_{k \ell}$, associated with the mode; see, e.g., Charpinet et al. 2000) and show their association with the observed frequencies. For each pair of associated frequencies, the quantities $\Delta P=P_{\mathrm{obs}}-P_{\mathrm{th}}, \Delta v=v_{\mathrm{obs}}-v_{\mathrm{th}}$, and $\Delta P / P=-\Delta v / v$ quantify the difference between the computed and measured values. For convenience, we also provide again, in these tables, the amplitude of the observed mode. Finally, we indicate within brackets the 11 additional frequencies that were not reported in Reed et al. (2010).

For model 1, the averaged relative dispersion is $\overline{|\Delta X / X|} \sim$ $0.35 \%(X=P$ or $v)$, which corresponds, on an absolute scale, to $\overline{|\Delta P|}=20.9 \mathrm{~s}$ and $\overline{|\Delta v|}=0.811 \tilde{\mathrm{Al}} \mathrm{l}^{\prime} \mu \mathrm{Hz}$. The standard deviations in these quantities are, respectively, $24.8 \mathrm{~s}$ and $0.787 \mu \mathrm{Hz}$. For model 2, the averaged relative dispersion is $\overline{|\Delta X / X|} \sim 0.39 \%$, corresponding to $\overline{|\Delta P|}=23.0 \mathrm{~s}$ and $\overline{|\Delta v|}=0.957 \mu \mathrm{Hz}$. The standard deviations are then, respectively, $24.2 \mathrm{~s}$ and $0.918 \mu \mathrm{Hz}$. Compared to previous analyses of $g$-mode sdB pulsators that were able to reproduce the frequencies with an average relative dispersion of $\sim 0.23 \%$ (see Van Grootel et al. 2010a,b), we find that the quality of the frequency fits achieved for KIC 02697388 shows a noticeable degradation. The larger number of modes that must be fitted simultaneously could possibly be one of the reasons why our best solutions for KIC02697388 do not as closely reproduce the observed spectrum as in the two other stars analysed thus far. However, it is also likely that our current inability to resolve properly all the pulsation modes contributes significantly to the larger dispersion between the best-fit models and the observations. The induced uncertainties in the accuracy of some of the frequencies used in this analysis and possible misidentifications of which component of a complex of modes is the $m=0$ mode (if rotation plays a role) could indeed interfere at a level close to the formal frequency resolution of the present data $(\sim 0.43 \mu \mathrm{Hz})$. Future time series obtained for this star with Kepler will undoubtedly improve the situation but, for the time being, this source of uncertainty remains sufficiently small to allow us to engage the detailed asteroseismic study of this star.

The 43 pulsations involved in our analysis are identified as low-degree $(\ell=1,2$, and 4$)$, intermediate-order $(k=-7$ through -64) g-modes ${ }^{2}$. We point out that almost all the very low amplitude frequencies above $500 \mu \mathrm{Hz}$ that were allowed to be either $\ell=1,2$, or 4 modes, turn out to be identified as $\ell=4$ modes. This confirms our initial intuition that these low amplitude frequencies could indeed be the dominant modes belonging to the $\ell=4$ series that, despite strong geometrical cancellation (see Fig. 11 and comments below), can emerge above the detection threshold of the present data (see Sect. 3.1). Looking at the amplitude distribution resulting from the mode identifications derived from each solution, we clearly find that, in both cases, the highest amplitude frequencies are preferentially associated with $\ell=1$ modes, as one would expect. To quantify this, we point out that, for model 1 (Table 3 ), eight frequencies were associated with $\ell=4$ modes. The average amplitude of these eight modes is $\bar{A}_{4,8 m}=0.0186 \%$. In comparison, we computed the average amplitudes, $\bar{A}_{1,8 m}$ and $\bar{A}_{2,8 m}$, of the eight strongest modes associated with $\ell=1$ and $\ell=2$, respectively. We find that $\bar{A}_{1,8 m}=0.1316 \%$ and $\bar{A}_{2,8 m}=0.0787 \%$. We therefore obtain the following average amplitude ratios of different degrees: $\bar{A}_{1,8 m} / \bar{A}_{2,8 m} \simeq 1.7, \bar{A}_{1,8 m} / \bar{A}_{4,8 m} \simeq 7.1$, and $\bar{A}_{2,8 m} / \bar{A}_{4,8 m} \simeq 4.2$. For model 2 (Table 4), the amplitude distribution is similar and we have $\bar{A}_{4,7 m}=0.0188 \%$ (with only seven modes associated with $\ell=4$ in this case), $\bar{A}_{1,7 m}=0.1362 \%$, and $\bar{A}_{2,7 m}=0.0983 \%$. The corresponding average amplitude ratios of different degrees become $\bar{A}_{1,7 m} / \bar{A}_{2,7 m} \simeq 1.4, \bar{A}_{1,7 m} / \bar{A}_{4,7 m} \simeq 7.2$, and $\bar{A}_{2,7 m} / \bar{A}_{4,7 m} \simeq$ 5.2. In both cases, the pronounced amplitude separation between $\ell=1,2$ and $\ell=4$ is qualitatively in line with the visibilities generally expected from computations (see, Fig. 11).

A more quantitative comparison of the averaged observed amplitude ratios with calculations remains however difficult for several reasons. Figure 11 shows the visibilities of $\ell=1,2,3$, and 4 modes expected for a star with parameters representative of KIC02697388. For $g$-modes in sdB stars, the light modulations are completely dominated by the temperature perturbation. These visibility curves were computed assuming the same intrinsic amplitude for the temperature perturbation and only $m=0$ modes were considered. The results are found to be independent of the pulsation frequency. The relative visibility of a mode clearly depends quite strongly on the (unknown) inclination angle of the star relative to the observer. The $\ell=3$ modes are the most affected by the geometrical cancellation effects except at very specific inclinations $\left(i \sim 30^{\circ}\right.$ and $\left.i \sim 70^{\circ}\right)$. There is a marked gap between $\ell=3,4$ and $\ell=1$ and 2 at nearly all viewing angles (except for $i \sim 55^{\circ}$ and $i \sim 90^{\circ}$ ). In the seismic analysis of KIC02697388, we assumed that all the observed frequencies were $m=0$ modes (as in Fig. 11), considering that, in the context of a star likely rotating relatively slowly, misidentifications of the $m$-index of the modes do not have a large impact on the asteroseismic solutions themselves. The visibility of the modes would however be greatly affected, depending on the inclination angle. An even more acute difficulty defeating meaningful quantitative comparisons of amplitude ratios is that the observed pulsation modes certainly do not have the same

2 We use, as a convenient convention, negative values for the radial order $k$ of $g$-modes, while $p$-modes are identified with positive values of $k$. 

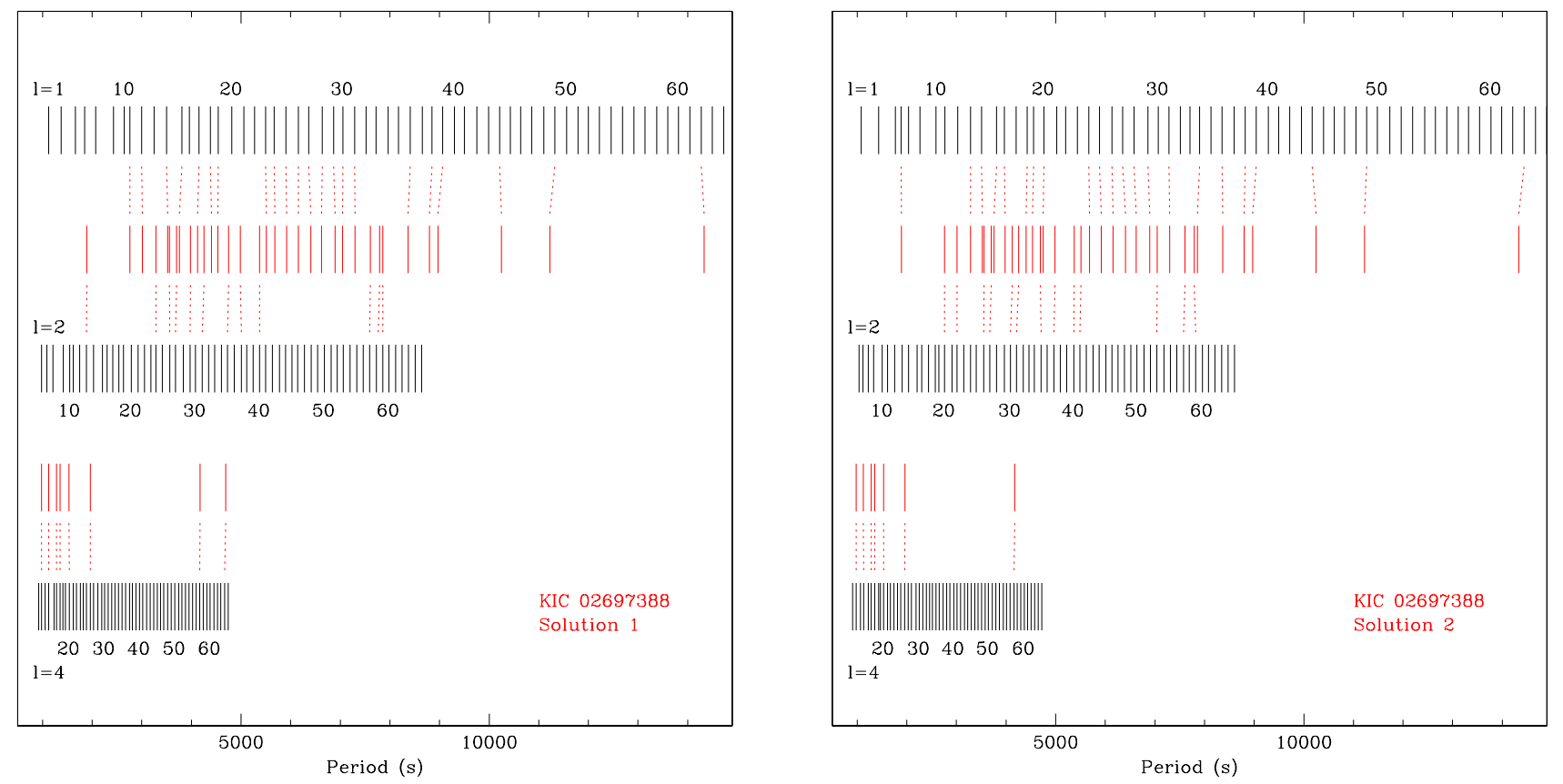

Fig. 10. Distribution of the observed periods of KIC02697388 (in red) compared to the $\ell=1, \ell=2$, and $\ell=4$ g-mode theoretical pulsation spectrum of the optimal models 1 (left panel) and 2 (right panel). The radial order $k$ of the computed modes is indicated for each series of degree.

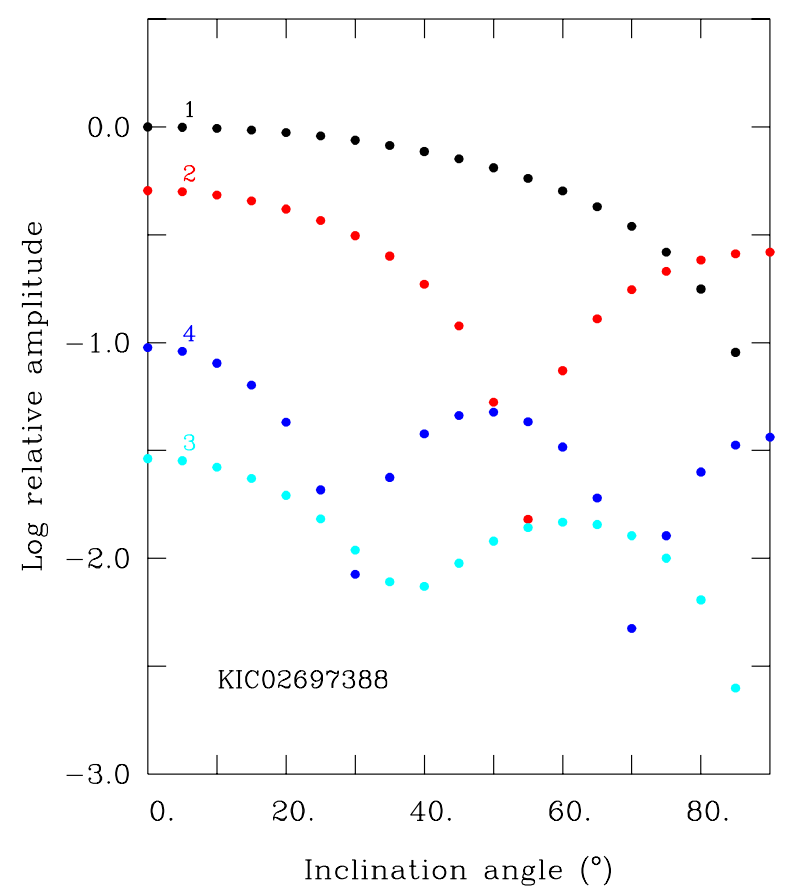

Fig. 11. Relative amplitude (in logarithmic scale) of $g$-mode oscillations as a function of the inclination angle and the degree $\ell$ (assuming that $m=0$ ). The values $\ell=1$ (black), 2 (red), 3 (cyan), and 4 (blue) are represented. These visibility functions are evaluated for a star with parameters representative of KIC02697388 and in the Kepler band-pass.

intrinsic amplitudes. It is therefore very difficult, in these conditions, to go beyond the qualitative argumentation provided so far.

\subsection{The high frequency oscillation}

An intriguing feature of KIC02697388 revealed by the analysis of the Kepler light curve is the presence of a very weak, isolated peak at $3805.94 \mu \mathrm{Hz}\left(262.75 \mathrm{~s} ; f_{38}\right.$ in Table 1). Our detailed asteroseismic study presented in the previous section demonstrate that this frequency is perfectly integrated into the best-fit solutions that emerge from this analysis and that it matches a low-order $(k=1)$ either radial $(\ell=0)$ or non-radial $(\ell=2)$ $p$-mode. These are the modes typically observed in $\mathrm{sdBV}_{\mathrm{r}}$ stars. A modulation at this short timescale, typical of the much hotter $p$-mode sdB pulsators, first raised skepticism regarding its true nature (real mode or instrumental artifact?). KIC02697388, according to our spectroscopy, is indeed one of the coolest known pulsating sdB stars and certainly among the last objects where we would expect to find acoustic modes. These modes are however extremely sensitive to the surface gravity $(\log g)$ of the star and the remarkable correspondence with the best-fit solutions uncovered provides strong evidence of a real $p$-mode.

There is more, however, which brings us to Fig. 2, where, as already mentioned, the position of KIC02697388 (green square with a cross) appears clearly separated from the other $\mathrm{sdBV}_{\mathrm{r}}$ and hybrid pulsators by more than $4000 \mathrm{~K}$. This figure also shows that there is no real conflict with theory on that surprising matter. The blue contours in Fig. 2 reproduce the expected instability region for the short period $p$-mode pulsations derived from nonadiabatic calculations (Charpinet et al. 2001). While all $\mathrm{sdBV}_{\mathrm{r}}$ and hybrid pulsators were, until now, always found within the three highest contours where the driving mechanism is most efficient to excite acoustic waves, the instability region actually covers a much larger region in the $\log g-T_{\text {eff }}$ plane. The red edge, in particular, is found to reach significantly cooler effective temperatures, including most of the known long period $g$-mode pulsators. KIC02697388, in particular, lies upon the edge of the instability region where models predict that one acoustic mode can 
Table 5. Nonadiabatic properties of low-order, low degree $p$-modes in the optimal models.

\begin{tabular}{|c|c|c|c|c|c|c|c|c|c|}
\hline \multirow[b]{2}{*}{$\ell$} & \multirow[b]{2}{*}{$k$} & \multicolumn{4}{|c|}{ Model solution 1} & \multicolumn{4}{|c|}{ Model solution 2} \\
\hline & & $\begin{array}{c}P_{\mathrm{obs}} \\
(\mathrm{s})\end{array}$ & $\begin{array}{l}P_{\text {th }} \\
(\mathrm{s})\end{array}$ & $\begin{array}{c}\sigma_{I} \\
\left(\mathrm{~s}^{-1}\right)\end{array}$ & $\begin{array}{c}\sigma_{I}^{\dagger} \\
\left(\mathrm{s}^{-1}\right)\end{array}$ & $\begin{array}{c}P_{\mathrm{obs}} \\
\text { (s) }\end{array}$ & $\begin{array}{l}P_{\text {th }} \\
(\mathrm{s})\end{array}$ & $\begin{array}{c}\sigma_{I} \\
\left(\mathrm{~s}^{-1}\right)\end{array}$ & $\begin{array}{c}\sigma_{I}^{\dagger} \\
\left(\mathrm{s}^{-1}\right)\end{array}$ \\
\hline$\overline{0}$ & 2 & $\ldots$ & 221.61 & $+3.819 \times 10^{-6}$ & $+2.642 \times 10^{-6}$ & $\ldots$ & 215.83 & $+4.394 \times 10^{-6}$ & $+3.708 \times 10^{-6}$ \\
\hline 0 & 1 & $\ldots$ & 269.50 & $-9.695 \times 10^{-9}$ & $-1.583 \times 10^{-7}$ & 262.75 & 262.50 & $+1.303 \times 10^{-7}$ & $-6.588 \times 10^{-9}$ \\
\hline 0 & 0 & $\ldots$ & 331.83 & $-2.790 \times 10^{-8}$ & $-2.902 \times 10^{-8}$ & $\ldots$ & 323.70 & $-2.320 \times 10^{-8}$ & $-2.749 \times 10^{-8}$ \\
\hline 1 & 3 & $\ldots$ & 219.72 & $+4.277 \times 10^{-6}$ & $+3.122 \times 10^{-6}$ & $\ldots$ & 214.08 & $+4.861 \times 10^{-6}$ & $+4.092 \times 10^{-6}$ \\
\hline 1 & 2 & $\ldots$ & 267.47 & $+2.732 \times 10^{-8}$ & $-1.255 \times 10^{-7}$ & $\ldots$ & 260.63 & $+1.744 \times 10^{-7}$ & $+2.604 \times 10^{-8}$ \\
\hline 1 & 1 & $\ldots$ & 327.27 & $-3.322 \times 10^{-8}$ & $-2.228 \times 10^{-8}$ & $\ldots$ & 319.48 & $-2.696 \times 10^{-8}$ & $-3.202 \times 10^{-8}$ \\
\hline 2 & 2 & $\ldots$ & 216.44 & $+5.043 \times 10^{-6}$ & $+3.851 \times 10^{-6}$ & $\ldots$ & 211.21 & $+5.555 \times 10^{-6}$ & $+3.751 \times 10^{-6}$ \\
\hline 2 & 1 & 262.75 & 262.55 & $+1.350 \times 10^{-7}$ & $-1.254 \times 10^{-8}$ & $\ldots$ & 256.25 & $+2.900 \times 10^{-7}$ & $+8.184 \times 10^{-8}$ \\
\hline 2 & 0 & $\ldots$ & 307.82 & $-2.112 \times 10^{-8}$ & $-6.608 \times 10^{-8}$ & $\ldots$ & 294.61 & $-6.471 \times 10^{-9}$ & $-4.148 \times 10^{-8}$ \\
\hline
\end{tabular}

Notes. $\uparrow$ Value obtained when the amount of levitating Fe is increased by $8 \%$.

still be excited. Looking into the details, we computed the nonadiabatic properties of the low-order low-degree $p$-modes in the two optimal solutions uncovered from the asteroseismic analysis. These can be found in Table 5 where, along with the computed period, $P_{\text {th }}$, we provide the nonadiabatic quantity $\sigma_{I}$ (i.e., the imaginary part of the eigen-frequency) indicating if a mode is stable $\left(\sigma_{I}>0\right)$ or driven $\left(\sigma_{I}<0\right)$. Table 5 shows that in both cases, the mode associated with the observed periodicity is found to be stable but lies right next to the excited mode. Driving this mode would clearly require only a very slight modification of the models. For instance, increasing the effective temperature by only $\sim 100 \mathrm{~K}$ is sufficient to excite this mode. Alternatively, we find that a slight increase of only $8 \%$ in the amount of iron in the driving region (see, e.g., Charpinet et al. 2009b) is sufficient to make this mode unstable, as indicated in Table 5. This result demonstrates that the presence of this high frequency is also consistent at the nonadiabatic level with the interpretation that it is a $p$-mode. The accumulation of evidence on this matter leads us to claim with confidence that the $262.75 \mathrm{~s}$ isolated signal observed in KIC02697388 is a $p$-mode driven by the usual $\kappa$-mechanism and that this star is a hybrid pulsator.

The position of the red edge is a direct function of the amount of iron supported by radiative levitation in the stellar envelope. The contours reproduced in Fig. 2 are obtained by assuming that the iron abundance has reached a diffusive equilibrium state between gravitational settling and radiative levitation. The distribution of short period $p$-mode pulsators (including hybrids), prior to the discovery of KIC02697388, suggested that the true instability region was possibly much narrower than expected under this assumption. A natural explanation was that the equilibrium abundance represents an upper limit that is not effectively reached because of competing processes. KIC02697388 somewhat challenges this idea since an iron abundance at diffusive equilibrium, at least, is needed to account for the presence of this pulsation mode in this star. This object expands considerably the observed red edge of the $p$-mode pulsations in sdB stars. KIC02697388 also raises the question of the presence of very low amplitude $p$-modes in other long period sdB pulsators, most of them being significantly hotter and therefore more prone, in principle, to excite acoustic waves. The outcome of the Kepler survey phase reported in Reed et al. (2010) and Baran et al. (2011) indeed provides indications that almost all other long period sdB pulsators may show such acoustic modes. The very low level of the amplitudes involved would naturally explains why these modes had never been detected before from the ground.

\subsection{Derived stellar parameters}

The asteroseismic analysis normally leads to the determination of the basic structural parameters of the scrutinized star. In the present situation, we do not have a single, clear-cut solution to propose for KIC02697388 but instead two solutions between which it is not possible to decide at this stage. We therefore derive two sets of parameters that reproduce equally well this star in terms of both spectroscopy and asteroseismology. These parameters are summarized in Table 6.

Estimating the $1 \sigma$ (internal) uncertainties associated with the primary quantities (those naturally derived from the asteroseismic analysis) is an important, but nontrivial task. Adopting a conservative approach, we adapted the recipe described in detail in Brassard et al. (2001) and Charpinet et al. (2005), but instead of using the maps intersecting with the solutions (Figs. 6 and 7), we considered the projected maps shown in Figs. 8 and 9. The projection of the $1 \sigma$ contour (i.e., the innermost white dashed contours shown in these figures) onto the various axis provides the $1 \sigma$ estimate of the corresponding parameter. Because the solutions (the yellow marks) are generally not well centered in the region defined by the $1 \sigma$ limit, the error estimates are given with differing plus and minus bounds. The uncertainties in the derived atmospheric parameters $T_{\text {eff }}$ and $\log g$ are obtained from the uncertainties in the primary quantities. To do so, we computed several models with parameters independently set to values corresponding to the various limits defining their $1 \sigma$ range. The differences observed in $T_{\text {eff }}$ and $\log g$ with the corresponding values obtained for the optimal model were then added quadratically, thus providing an estimate of the global uncertainties in these quantities. A set of secondary parameters (stellar radius $R$, luminosity $L$, age) is also derived on the basis of the primary parameters. Their associated errors are estimated in the same way as the uncertainties obtained for the effective temperature and surface gravity, i.e., by computing their values at the boundaries of the $1 \sigma$ domain and adding the differences with the optimal model quadratically. To evaluate the age associated with each solution, we used new evolutionary models that incorporate the same input physics employed in our third generation static structures. These evolutionary calculations are described in a forthcoming paper (Brassard et al. 2011, in prep.). In particular, these models include diffusion, gravitational settling, radiative levitation, core overshooting, and a time-dependent treatment for the convection. They also provide a treatment for the coupling between nuclear reactions and diffusion. To produce larger cores that can 
Table 6. Structural and core parameters inferred for KIC02697388 for the two solutions.

\begin{tabular}{lcc}
\hline \hline Quantity & \multicolumn{2}{c}{ Estimated values } \\
& solution 1 & solution 2 \\
\hline$T_{\text {eff }}(\mathrm{K})$ & \multicolumn{2}{c}{$25395 \pm 227^{\dagger}$} \\
& ${ }^{\dagger} 25622_{-350}^{+490}$ & ${ }^{\dagger} 25555_{-560}^{+480}$ \\
$\log g(\mathrm{cgs})$ & \multicolumn{2}{c}{$5.500 \pm 0.031^{\dagger}$} \\
& $5.489_{-0.036}^{+0.029}$ & ${ }^{\ddagger} 5.499_{-0.051}^{+0.047}$ \\
\hline$M_{*} / M_{\odot}$ & $0.463_{-0.008}^{+0.010}$ & $0.452_{-0.005}^{+0.018}$ \\
$\log \left(M_{\text {env }} / M_{*}\right)$ & $-2.30_{-0.06}^{+0.03}$ & $-2.35_{-0.02}^{+0.07}$ \\
$\log \left(1-M_{\text {core }} / M_{*}\right)$ & $-0.39_{-0.02}^{+0.01}$ & $-0.30_{-0.01}^{+0.03}$ \\
$M_{\text {core }} / M_{\odot}$ & $0.274_{-0.010}^{+0.008}$ & $0.225_{-0.016}^{+0.011}$ \\
$X_{\text {core }}(\mathrm{C}+\mathrm{O})$ & $0.18_{-0.03}^{+0.06}$ & $0.27_{-0.12}^{+0.07}$ \\
\hline Age $(\mathrm{Myr})^{*}$ & $40.8 \pm 1.0$ & $53.9 \pm 1.0$ \\
$R / R_{\odot}\left(M_{*}, g\right)$ & $0.203_{-0.007}^{+0.009}$ & $0.198_{-0.011}^{+0.013}$ \\
$L / L_{\odot}\left(T_{\text {eff }}, R\right)$ & $16.0_{-1.4}^{+1.9}$ & $15.1_{-2.0}^{+2.3}$ \\
\hline$M_{V}\left(g, T_{\text {eff }}, M_{*}\right)$ & $4.394 \pm 0.133$ & $4.450 \pm 0.187$ \\
$V$ & $15.234 \pm 0.021$ \\
$B-V$ & $-0.164 \pm 0.030$ \\
$E(B-V)$ & $0.057 \pm 0.030$ & $0.056 \pm 0.030$ \\
$A_{V}$ & $0.182 \pm 0.096$ & $0.179 \pm 0.096$ \\
$d\left(V, M_{V}, A_{V}\right)(\mathrm{pc})$ & $1355 \pm 144$ & $1321 \pm 173$ \\
\hline
\end{tabular}

Notes. ${ }^{\ddagger}$ From asteroseismology; ${ }^{\dagger}$ from spectroscopy; ${ }^{*}$ from the zero age extreme horizontal branch (ZAEHB).

match those suggested by our asteroseismic results, an ad-hoc turbulent diffusion term was added. While the eventual origin of this contribution has yet to be clarified, we note that differential rotation, for instance, could produce this additional mixing. These evolutionary models provide the closest counterparts to the static structures identified from the asteroseismic analysis. We point out that the age of the star depends on both the composition (i.e., the amount of helium burnt) and the size of its core. In this advanced evolutionary stage, a larger core requires more time to burn a given fraction (in mass) of the available helium. As a comparison, sequences computed without this extra mixing term and therefore having a smaller core would lead to age estimates of $21.5 \mathrm{Myr}$ and $37.1 \mathrm{Myr}$ for models \#1 and \#2, respectively. Finally, we point out that all the uncertainties quoted in Table 6 are statistical ones, with the understanding that uncertainties of systematic effects caused by imperfections in the models are not considered here, because these are by definition difficult to estimate.

A third set of parameters (absolute magnitude $M_{V}$, reddening index $E(B-V)$, extinction between Earth and the star, $A_{V}$, and distance from Earth $d$ ) can be deduced from the primary and secondary quantities by further exploiting our model atmospheres. To obtain these quantities, we have to exploit the wide band photometry available for KIC02697388. The most accurate measurements are from the SDSS-DR7 catalog, in the ugriz system $: u=15.061 \pm 0.004, g=15.003 \pm 0.003, r=15.419 \pm 0.003$, $i=15.685 \pm 0.004, z=15.925 \pm 0.007$. We used the transformations from Jester et al. (2005) to evaluate the corresponding Johnson $V$ magnitude and the apparent $(B-V)$ color index of KIC02697388 (see Table 6). From the unreddened color index $(B-V)_{0}$ derived from the spectral energy distribution expected from the parameters associated with solution 1 and 2 , we compute the reddening index, $E(B-V)$, and the extinction coefficient, $A_{V}$. The distance from Earth, $d$, then readily follows. We point out that it is also possible to work directly with the ugriz system without relying on transformations. Following the same procedure and making use of the atmospheric parameters derived from spectroscopy and the stellar radius obtained from solution 1, we evaluate a reddening index $E(g-r)=0.058 \pm 0.027$ and an absorption coefficient $A_{r}=0.154 \pm 0.072$. The distance (corrected from extinction) obtained with the $g$-band is then $d=$ $1351 \pm 131 \mathrm{pc}$. Using the $r$-band, we derive $d=1351 \pm 128 \mathrm{pc}$. Overall, there is excellent agreement among the values obtained for the distance, with and without using the Jester et al. (2005) transformations.

Interestingly, we find that the two separate solutions proposed for KIC02697388 converge toward nearly identical values for several of the parameters of the star. Within the estimated errors, the mass, radius, luminosity, effective temperature, and surface gravity of the two solutions do not differ significantly. Other quantities relying on these parameters, i.e., absolute magnitude, reddening index, extinction coefficient, and distance from Earth, therefore also converge for practically the same values. Hence, these quantities, despite the remaining ambiguity concerning the true model solution, have values that are securely constrained. We find in particular that the inferred mass for KIC02697388 is close to the canonical value expected for hot B subdwarfs (Dorman et al. 1993; Han et al. 2002, 2003). The hydrogenrich envelope mass is also tightly constrained despite having two model solutions. The inferred values do not strongly differ from one model to the other. The envelope is found to be thicker than envelopes measured in other pulsating sdB stars, including the two other $g$-mode pulsators studied thus far (Van Grootel et al. 2010a,b). This result is in line with the expected correlation between $T_{\text {eff }}$ and $M_{\text {env }}$ (cooler extreme horizontal branch stars should have thicker envelopes), considering that KIC02697388 is the coolest sdB star analysed with asteroseismology, thus far.

The other parameters related to the helium-burning core are of particular interest. It was hoped that g-modes in sdB stars can probe the properties of the core, and they do, as first demonstrated by Van Grootel et al. (2010a). However, in the present case, owing to the existence of two solutions that we cannot separate at this stage, there remain relatively large uncertainties in the values defining the size and composition of the $\mathrm{He}$ burning core. Despite this limitation, the carbon-enriched core is found to be rather massive, although it has burned less than $\sim 34 \%$ of its helium nuclear fuel (model solution 2 corresponding to the most advanced stage). In comparison, the fully mixed core in standard evolutionary models only reaches $\sim 0.14 M_{\odot}$ at these stages. This finding is in line with the trends already noted for KPD1943+4058 (Van Grootel et al. 2010a) and KPD 06290016 (Van Grootel et al. 2010b). Therefore, this size is significantly above that expected from evolutionary tracks computed by, e.g., Dorman et al. (1993). These authors, however, defined the convective core using the Schwarzschild criterion and did not consider overshooting, while the chemical transition $\mathrm{He} / \mathrm{C} / \mathrm{O}-\mathrm{He}$ defines its boundary in our models. Our measurement may therefore be the signature of an extended core due to, e.g., overshooting, whereby processed material $(\mathrm{C}+\mathrm{O})$ is carried out by momentum beyond the convection zone.

\section{Summary and conclusion}

We have presented the first detailed asteroseismic sounding of the hot B subdwarf $g$-mode pulsator KIC02697388 based on the high quality, nearly continuous time-series photometry provided by the Kepler satellite. We reanalysed in detail the 27day-longdata from the run Q2.3 available for that star (see, 
Østensen et al. 2010; Reed et al. 2010) and extracted 59 peaks judged to be both significant and unassociated with known instrumental artifacts (see Table 1; online version only). In our analysis of the light curve, the high frequency peak reported in Reed et al. (2010) was also recovered at a quite significant level $(S / N \sim 5.7)$. This peak is of particular interest because its associated timescale (a period of $262.75 \mathrm{~s}$ ) is typical of acoustic mode oscillations (see below).

Before interpreting the pulsations of KIC02697388, we performed a detailed analysis of the spectroscopic properties of this star. A new, high signal-to-noise, low resolution spectrum of KIC02697388 was obtained and fitted with synthetic spectra computed from NLTE H/He model atmospheres assuming a representative (non-solar) heavy-element composition inspired by Blanchette et al. (2008). This led us to determine the atmospheric parameters for KIC02697388 $T_{\mathrm{eff}}=25395 \pm 227 \mathrm{~K}$, $\log g=5.500 \pm 0.031$, and $\log N(\mathrm{He}) / N(\mathrm{H})=-2.767 \pm 0.122$. The derived atmospheric parameters indicate that KIC02697388 lies on the cool side of the region in the $\log g-T_{\text {eff }}$ plane where the $g$-mode sdB pulsators are found (see Fig. 2). In this context, the presence of a high frequency modulation becomes intriguing as this star lies far away from the region where hybrid pulsators (showing both $p$ - and $g$-modes) had been found until now (typically near $T_{\text {eff }} \sim 30000 \mathrm{~K}$ for temperatures derived using NLTE model atmospheres).

The seismic sounding was conducted using our so-called 3rd generation $(3 \mathrm{G})$ models, which are appropriate for the quantitative interpretation of $g$-mode pulsations in sdB stars. We explored the vast model parameter space with our forward modelling approach (see, e.g., Charpinet et al. 2008) and identified the two families of solutions that most closely match the observed frequencies equally well and agree with the constraints provided by the spectroscopically determined atmospheric parameters of KIC02697388.

At this stage, we are unable to distinguish the two solutions uncovered in terms of quality of fit or based on other arguments. Both models match the observed frequencies used in the analysis equally well, with an average dispersion of $\sim 0.35 \%(0.81 \mu \mathrm{Hz})$ and $\sim 0.39 \%(0.96 \mu \mathrm{Hz})$, respectively. This level of accuracy is slightly worse than what we were able to achieve for the two other $g$-mode sdB pulsators analysed thus far, KPD 1943+4058 and KPD 0629-0016, where the typical average dispersion obtained between modeled and observed frequencies was $\sim 0.23 \%$ $(0.70 \mu \mathrm{Hz}$; Van Grootel et al. 2010a,b). We attribute this difference to unresolved fine structure (possibly due to rotation) in the frequency spectrum of KIC02697388 that, for some modes, increases the uncertainty in their frequencies. Beyond this additional source of error, we recall that an important fraction of the current discrepancies between the models and the observations certainly resides in inadequacies in the modelling. These are expected and obviously constitute one of the major reasons to carry out such detailed asteroseismic studies for guiding current efforts towards developing more realistic stellar models.

The mode identifications obtained as a result of the forward modelling procedure provide, in both cases, a highly consistent picture of the nature of the modes detected in KIC02697388. The frequencies are interpreted in terms of superimposed series of mid-order $(|k| \sim 7-64)$ gravity modes of degree $\ell=1,2$, and 4 , respectively. We have found a hierarchy in amplitude for the fitted/identified frequencies that is consistent with the distribution of apparent amplitudes (on average) expected from the attenuation factors caused by geometrical cancellation. The mode distribution is also in line with the nonadiabatic calculations presented in Fontaine et al. (2003) that show a shift of the driven $g$-modes to higher frequencies (shorter periods) as the degree increases (see their Fig. 9).

For the two asteroseismic solutions uncovered, the high frequency detected in the Kepler light curve can be naturally associated with a low-order $(k=1)$, low-degree (either $\ell=0$ or 2) $p$-mode. Low order, low degree acoustic modes are typically those being excited and observed in the short period sdB pulsators. Despite being significantly cooler than the other sdB stars featuring these kind of rapid oscillations, we demonstrated that KIC02697388 is not in conflict with the nonadiabatic theory predicting the excitation of $p$-modes in sdB stars. We have demonstrated that this star lies on the edge of the expected instability region derived from the assumption that the iron abundance in the stellar envelope has reached its equilibrium state from the competition of radiative levitation against gravitational sedimentation (Fig. 2). We demonstrated that the acoustic modes predicted to be excited in the two optimal models uncovered include (within the model uncertainties) the high frequency mode effectively observed in KIC02697388 (Table 5). All these converging elements constitute strong evidence that this isolated frequency is indeed a driven $p$-mode occurring in a $\mathrm{sdB}$ star that is significantly cooler than any other sdB exhibiting these kinds of pulsations. This finding indicates that the observed red edge of the $p$-mode instability region is significantly cooler than believed so far. The very small amplitude of the $p$-mode seen in KIC02697388 (only 156 ppm and 5.7 times above the noise in the Kepler data), if representative of the amplitudes of acoustic waves in this part of the $\log g-T_{\text {eff }}$ plane, could of course naturally explain why these modes have never been detected previously in cool sdB stars.

At this stage, our data for KIC02697388 does not allow us to identify a single solution for its oscillation spectrum. It is therefore impossible to propose a unique determination of its structural parameters. The values associated with each solution are summarized in Table 6. Despite this remaining ambiguity, we nonetheless find that the values derived for several parameters (i.e., the total mass, $M_{*}$, the envelope mass, $M_{\text {env }}$, the radius, $R$, and the luminosity, $L$ ) are very similar and therefore constitute robust estimates for some of the properties of KIC02697388, independent of which solution turns out to be the "real" one. The main differences between the two families of models occur at the level of the composition of the mixed core, $X_{\text {core }}(\mathrm{C}+\mathrm{O})$, and in the evaluation of its size, $\log \left(1-M_{\text {core }} / M_{*}\right)$. In any case, KIC02697388 is found to be a relatively young sdB star (younger than $~ 55$ Myr since the ZAEHB) having burnt less than $\sim 34 \%$ of its helium (in mass). In line with previous findings for the stars KPD 1943+4058 (Van Grootel et al. 2010a) and KPD 0629-0016 (Van Grootel et al. 2010b), we also determined - although we cannot provide a unique value for this parameter - that the size of the mixed core is significantly larger than the size normally obtained by the Schwarzschild criterion defining the inner convective core. This suggests once again that extra mixing (caused by overshooting or differential rotation, for instance) has taken place early in the evolution of the He core in these stars, such that the outer boundary of the $\mathrm{He} / \mathrm{C} / \mathrm{O}$ region (the quantity that we estimate here) has been pushed outward. This trend appears to be confirmed now that three g-mode $\mathrm{sdB}$ pulsators have thus far been analysed by asteroseismology. Additional studies of this type for other identified $g$-mode pulsators should shed further light on this fascinating issue.

The present analysis of KIC02697388 was based on the first run of time series photometry acquired by the Kepler mission on this star. More data are currently being collected and are expected to provide a major improvement to the frequency 
resolution (due to the longer coverage). This should allow us to properly resolve the various complexes of close frequencies that have been observed in the light curve analysis of KIC02697388. If generated by rotational splitting, this fine structure would hold the key to the detailed study of the internal dynamics of this star. It could also help us to identify some of the observed modes more precisely, possibly allowing us to discriminate between the two currently proposed seismic solutions. We are therefore looking forward to exploiting new data from Kepler on the remarkable sdB star that is KIC02697388.

Acknowledgements. S.C. and V.V.G. thank the Programme National de Physique Stellaire (PNPS, CNRS/INSU, France) for financial support. Numerical experiments presented in this paper were carried out using the Grid'5000 experimental testbed, an initiative from the French Ministry of Research through the ACI GRID incentive action, INRIA, CNRS and RENATER, and other contributing partners (see http://www.grid5000.fr). G.F. acknowledges the support of the NSERC of Canada and the contribution of the Canada Research Chair Program. The authors gratefully acknowledge the Kepler team and everyone who has contributed to making this mission possible. Funding for the Kepler mission is provided by NASA's Science Mission Directorate.

\section{References}

Baglin, A., Auvergne, M., Barge, P., et al. 2006, in ESA SP-1306, ed. M. Fridlund, A. Baglin, J. Lochard, \& L. Conroy, 33

Baran, A., Oreiro, R., Pigulski, A., et al. 2009, MNRAS, 392, 1092

Baran, A., Kawaler, S. D., Reed, M. D., et al. 2011, MNRAS, in press [arXiv: 1103.1666]

Blanchette, J., Chayer, P., Wesemael, F., et al. 2008, ApJ, 678, 1329

Brassard, P., \& Fontaine, G. 2008, in Hot Subdwarf Stars and Related Objects, ed. U. Heber, C. S. Jeffery, \& R. Napiwotzki, ASP Conf. Ser., 392, 261 Brassard, P., \& Fontaine, G. 2009, J. Phys. Conf. Ser., 172, 2016

Brassard, P., Fontaine, G., Billères, M., et al. 2001, ApJ, 563, 1013

Brassard, P., Fontaine, G., Chayer, P., \& Green, E.M. 2010, 17th European White Dwarf Workshop, ed. K. Werner, \& T. Rauch, AIP Conf. Proc., 1273, 259

Charpinet, S., Fontaine, G., Brassard, P., \& Dorman, B. 1996, ApJ, 471, L103

Charpinet, S., Fontaine, G., Brassard, P., et al. 1997, ApJ, 483, L123

Charpinet, S., Fontaine, G., Brassard, P., \& Dorman, B. 2000, ApJS, 131, 223

Charpinet, S., Fontaine, G., \& Brassard, P. 2001, PASP, 113, 775

Charpinet, S., Fontaine, G., Brassard, P., Green, E. M., \& Chayer, P. 2005, A\&A, 437, 575

Charpinet, S., van Grootel, V., Reese, D., et al. 2008, A\&A, 489, 377

Charpinet, S., Brassard, P., Fontaine, G., et al. 2009a, in AIP Conf. Ser., ed. J. A. Guzik, \& P. A. Bradley, 1170, 585

Charpinet, S., Fontaine, G., \& Brassard, P. 2009b, A\&A, 493, 595

Charpinet, S., Green, E. M., Baglin, A., et al. 2010, A\&A, 516, L6

D’Cruz, N. L., Dorman, B., Rood, R. T., \& O’Connell, R. W. 1996, ApJ, 466, 359
Deeming, T. J. 1975, Ap\&SS, 36, 137

Dorman, B., Rood, R. T., \& O'Connell, R. W. 1993, ApJ, 419, 596 Fontaine, G., Brassard, P., Charpinet, S., et al. 2003, ApJ, 597, 518

Geier, S., Edelmann, H., Heber, U., \& Morales-Rueda, L. 2009a, ApJ, 702, L96

Geier, S., Heber, U., Edelmann, H., et al. 2009b, J. Phys. Conf. Ser., 172, 012008 Gilliland, R. L., Brown, T. M., Christensen-Dalsgaard, J., et al. 2010, PASP, 122, 131

Green, E. M., Fontaine, G., Reed, M. D., et al. 2003, ApJ, 583, L31

Han, Z., Podsiadlowski, P., Maxted, P. F. L., Marsh, T. R., \& Ivanova, N. 2002, MNRAS, 336, 449

Han, Z., Podsiadlowski, P., Maxted, P. F. L., \& Marsh, T. R. 2003, MNRAS, 341, 669

Heber, U. 2009, ARA\&A, 47, 211

Hubeny, I., \& Lanz, T. 1995, ApJ, 439, 875

Jeffery, C. S., \& Saio, H. 2006a, MNRAS, 371, 659

Jeffery, C. S., \& Saio, H. 2006b, MNRAS, 372, L48

Jeffery, C. S., \& Saio, H. 2007, MNRAS, 378, 379

Jenkins, J. M., Caldwell, D. A., Chardrasekaran, H., et al. 2010, ApJ, 713, L87

Jester, S., Schneider, D. P., Richards, G. T., et al. 2005, AJ, 130, 873

Kawaler, S. D., Reed, M. D., Østensen, R. H., et al. 2010a, MNRAS, 409, 1509

Kawaler, S. D., Reed, M. D., Quint, A. C., et al. 2010b, MNRAS, 409, 1487

Kilkenny, D., Koen, C., O’Donoghue, D., \& Stobie, R. S. 1997, MNRAS, 285, 640

Lanz, T., \& Hubeny, I. 1995, ApJ, 439, 905

Latour, M., Fontaine, G., Brassard, P., Chayer, P., \& Green, E. M. 2010, 17th European White Dwarf Workshop, ed. K. Werner, \& T. Rauch, AIP Conf. Proc., 1273, 255

Lee, J. W., Kim, S., Kim, C., et al. 2009, AJ, 137, 3181

Marcy, G., Butler, R. P., Fischer, D., et al. 2005, Progr. Theor. Phys. Suppl., 158, 24

Østensen, R. H., Silvotti, R., Charpinet, S., et al. 2010, MNRAS, 409, 1470

Østensen, R. H., Silvotti, R., Charpinet, S., et al. 2011, MNRAS, in press [arXiv: 1101.4150$]$

Randall, S. K., Fontaine, G., Brassard, P., \& Bergeron, P. 2005, ApJS, 161, 456

Randall, S. K., Fontaine, G., Green, E. M., et al. 2006a, ApJ, 643, 1198

Randall, S. K., Green, E. M., Fontaine, G., et al. 2006b, ApJ, 645, 1464

Randall, S. K., van Grootel, V., Fontaine, G., Charpinet, S., \& Brassard, P. 2009, A\&A, 507, 911

Reed, M. D., Kawaler, S. D., Ostensen, R. H., et al. 2010, MNRAS, 409, 1496

Reed, M. D., Baran, A., Quint, A. C., et al. 2011, MNRAS, in press [arXiv: 1102.4286]

Saffer, R. A., Bergeron, P., Koester, D., \& Liebert, J. 1994, ApJ, 432, 351

Scargle, J. D. 1982, ApJ, 263, 835

Schuh, S., Huber, J., Dreizler, S., et al. 2006, A\&A, 445, L31

Silvotti, R., Schuh, S., Janulis, R., et al. 2007, Nature, 449, 189

Soker, N. 1998, AJ, 116, 1308

Stoughton, C., Lupton, R. H., Bernardi, M., et al. 2002, AJ, 123, 485

Udry, S., \& Santos, N. C. 2007, ARA\&A, 45, 397

Van Grootel, V., Charpinet, S., Fontaine, G., \& Brassard, P. 2008a, A\&A, 483, 875

Van Grootel, V., Charpinet, S., Fontaine, G., et al. 2008b, A\&A, 488, 685

Van Grootel, V., Charpinet, S., Fontaine, G., et al. 2010a, ApJ, 718, L97

Van Grootel, V., Charpinet, S., Fontaine, G., Green, E. M., \& Brassard, P. 2010b, A\&A, 524, 63

Pages 17 to 20 are available in the electronic edition of the journal at http://www . aanda. org 
S. Charpinet et al.: Deep asteroseismic sounding of the sdB pulsator KIC02697388 from Kepler time series photometry

Table 3. Mode identification and details of the frequency fit obtained from the optimal model solution 1.

\begin{tabular}{|c|c|c|c|c|c|c|c|c|c|c|c|c|}
\hline$\ell$ & $k$ & $\begin{array}{c}v_{\mathrm{obs}} \\
(\mu \mathrm{Hz})\end{array}$ & $\begin{array}{c}v_{\text {th }} \\
(\mu \mathrm{Hz})\end{array}$ & $\begin{array}{c}P_{\text {obs }} \\
(\mathrm{s})\end{array}$ & $\begin{array}{l}P_{\text {th }} \\
(\mathrm{s})\end{array}$ & $\begin{array}{c}\log E \\
(\mathrm{erg})\end{array}$ & $C_{k \ell}$ & $\begin{array}{c}\Delta P / P \\
(\%)\end{array}$ & $\begin{array}{l}\Delta P \\
(\mathrm{~s})\end{array}$ & $\begin{array}{c}\Delta v \\
(\mu \mathrm{Hz})\end{array}$ & $\begin{array}{c}\text { Ampl. } \\
(\%)\end{array}$ & Id. \\
\hline 1 & -10 & & 378.221 & & 2643.95 & 45.541 & 0.4796 & & & & & \\
\hline 1 & -11 & 362.702 & 362.510 & 2757.09 & 2758.54 & 45.498 & 0.4805 & -0.05 & -1.46 & +0.191 & 0.0337 & $f_{22}$ \\
\hline 1 & -12 & 332.366 & 333.453 & 3008.73 & 2998.92 & 45.470 & 0.4878 & +0.33 & +9.81 & -1.087 & 0.1023 & $f_{8}$ \\
\hline 1 & -13 & $\ldots$ & 307.716 & $\ldots$ & 3249.75 & 44.967 & 0.4866 & $\ldots$ & $\ldots$ & $\ldots$ & $\ldots$ & \\
\hline 1 & -14 & 284.310 & 285.810 & 3517.28 & 3498.82 & 45.024 & 0.4912 & +0.52 & +18.46 & -1.500 & 0.0622 & $f_{11}$ \\
\hline 1 & -15 & 266.164 & 262.794 & 3757.09 & 3805.26 & 44.360 & 0.4869 & -1.28 & -48.17 & +3.370 & 0.0553 & $f_{13}$ \\
\hline 1 & -16 & $\ldots$ & 252.511 & $\ldots$ & 3960.22 & 44.210 & 0.4876 & $\ldots$ & $\ldots$ & $\ldots$ & $\ldots$ & \\
\hline 1 & -17 & 242.449 & 240.991 & 4124.59 & 4149.53 & 44.672 & 0.4920 & -0.60 & -24.94 & +1.457 & 0.0473 & $f_{15}$ \\
\hline 1 & -18 & [227.150] & 228.182 & [4402.38] & 4382.47 & 43.955 & 0.4915 & {$[+0.45]$} & {$[+19.91]$} & {$[-1.032]$} & {$[0.0163]$} & {$\left[f_{36}\right]$} \\
\hline 1 & -19 & 220.457 & 220.225 & 4536.03 & 4540.80 & 43.588 & 0.4884 & -0.11 & -4.77 & +0.232 & 0.0333 & $f_{23}$ \\
\hline 1 & -20 & $\ldots$ & 207.728 & $\ldots$ & 4813.99 & 43.974 & 0.4947 & $\ldots$ & $\ldots$ & $\ldots$ & $\ldots$ & \\
\hline 1 & -21 & $\ldots$ & 197.764 & $\ldots$ & 5056.53 & 43.646 & 0.4923 & $\ldots$ & $\ldots$ & $\ldots$ & $\ldots$ & \\
\hline 1 & -22 & $\ldots$ & 189.632 & $\ldots$ & 5273.36 & 43.880 & 0.4943 & $\ldots$ & $\ldots$ & $\ldots$ & $\ldots$ & \\
\hline 1 & -23 & 181.614 & 181.895 & 5506.18 & 5497.67 & 43.952 & 0.4945 & +0.15 & +8.51 & -0.281 & 0.0616 & $f_{12}$ \\
\hline 1 & -24 & 176.060 & 176.341 & 5679.88 & 5670.83 & 43.912 & 0.4947 & +0.16 & +9.04 & -0.281 & 0.0342 & $f_{20}$ \\
\hline 1 & -25 & 168.868 & 169.448 & 5921.78 & 5901.51 & 43.969 & 0.4957 & +0.34 & +20.27 & -0.580 & 0.2539 & $f_{1}$ \\
\hline 1 & -26 & [162.482] & 162.371 & [6154.54] & 6158.75 & 43.668 & 0.4945 & {$[-0.07]$} & {$[-4.21]$} & {$[+0.111]$} & {$[0.0205]$} & {$\left[f_{31}\right]$} \\
\hline 1 & -27 & 156.163 & 157.161 & 6403.57 & 6362.92 & 43.736 & 0.4956 & +0.63 & +40.65 & -0.998 & 0.1440 & $f_{5}$ \\
\hline 1 & -28 & 150.929 & 150.741 & 6625.65 & 6633.89 & 43.900 & 0.4968 & -0.12 & -8.24 & +0.188 & 0.1149 & $f_{6}$ \\
\hline 1 & -29 & 145.023 & 145.607 & 6895.45 & 6867.81 & 43.526 & 0.4954 & +0.40 & +27.63 & -0.584 & 0.1652 & $f_{3}$ \\
\hline 1 & -30 & 141.994 & 141.854 & 7042.57 & 7049.50 & 43.519 & 0.4958 & -0.10 & -6.93 & +0.140 & 0.0227 & $f_{28}$ \\
\hline 1 & -31 & 137.073 & 137.188 & 7295.39 & 7289.27 & 43.615 & 0.4968 & +0.08 & +6.12 & -0.115 & 0.0340 & $f_{21}$ \\
\hline 1 & -32 & $\ldots$ & 133.032 & $\ldots$ & 7517.01 & 43.342 & 0.4961 & $\ldots$ & $\ldots$ & $\ldots$ & $\ldots$ & \\
\hline 1 & -35 & $\ldots$ & 122.340 & $\ldots$ & 8173.95 & 43.132 & 0.4964 & $\ldots$ & $\ldots$ & $\ldots$ & $\ldots$ & \\
\hline 1 & -36 & 119.546 & 118.895 & 8364.96 & 8410.78 & 43.258 & 0.4973 & -0.55 & -45.82 & +0.651 & 0.0291 & $f_{24}$ \\
\hline 1 & -37 & $\ldots$ & 115.533 & $\ldots$ & 8655.53 & 43.056 & 0.4967 & $\ldots$ & $\ldots$ & $\ldots$ & $\ldots$ & \\
\hline 1 & -38 & 113.647 & 113.056 & 8799.15 & 8845.14 & 43.056 & 0.4969 & -0.52 & -45.99 & +0.591 & 0.1489 & $f_{4}$ \\
\hline 1 & -39 & 111.481 & 110.313 & 8970.10 & 9065.14 & 43.245 & 0.4977 & -1.06 & -95.04 & +1.169 & 0.0444 & $f_{16}$ \\
\hline 1 & -40 & $\ldots$ & 107.554 & $\ldots$ & 9297.69 & 43.027 & 0.4973 & $\ldots$ & $\ldots$ & $\ldots$ & $\ldots$ & \\
\hline 1 & -43 & $\ldots$ & 100.070 & $\ldots$ & 9992.98 & 42.971 & 0.4977 & $\ldots$ & $\ldots$ & $\ldots$ & $\ldots$ & \\
\hline 1 & -44 & 97.565 & 97.945 & 10249.60 & 10209.83 & 42.999 & 0.4978 & +0.39 & +39.76 & -0.380 & 0.0276 & $f_{26}$ \\
\hline 1 & -45 & $\ldots$ & 95.924 & $\ldots$ & 10424.90 & 43.037 & 0.4979 & $\ldots$ & $\ldots$ & $\ldots$ & $\ldots$ & \\
\hline 1 & -48 & $\ldots$ & 90.079 & & 11101.36 & 42.943 & 0.4981 & $\ldots$ & $\ldots$ & $\ldots$ & $\ldots$ & \\
\hline 1 & -49 & 89.115 & 88.283 & 11221.43 & 11327.16 & 42.949 & 0.4982 & -0.94 & -105.73 & +0.832 & 0.0405 & $f_{17}$ \\
\hline 1 & -50 & $\ldots$ & 86.475 & $\ldots$ & 11564.09 & 43.041 & 0.4985 & $\ldots$ & $\ldots$ & $\ldots$ & $\ldots$ & \\
\hline 1 & -61 & $\ldots$ & 71.204 & $\ldots$ & 14044.20 & 42.882 & 0.4989 & $\ldots$ & & & $\ldots$ & \\
\hline 1 & -62 & [69.770] & 70.051 & [14332.75] & 14275.32 & 42.803 & 0.4988 & {$[+0.40]$} & {$[+57.42]$} & {$[-0.281]$} & [0.0225] & {$\left[f_{30}\right]$} \\
\hline 1 & -63 & $\ldots$ & 68.974 & $\ldots$ & 14498.32 & 42.779 & 0.4988 & $\ldots$ & $\ldots$ & $\ldots$ & $\ldots$ & \\
\hline 2 & 2 & & 4623.089 & & 216.31 & 41.139 & 0.0010 & $\cdots$ & 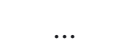 & & & \\
\hline 2 & 1 & 3805.944 & 3809.371 & 262.75 & 262.51 & 41.502 & 0.0041 & +0.09 & +0.24 & -3.427 & 0.0156 & $f_{38}$ \\
\hline 2 & 0 & $\ldots$ & 3226.526 & $\ldots$ & 309.93 & 42.659 & 0.0575 & $\ldots$ & $\ldots$ & $\ldots$ & $\ldots$ & \\
\hline 2 & -12 & $\ldots$ & 573.378 & $\ldots$ & 1744.05 & 45.440 & 0.1548 & & & & & \\
\hline 2 & -13 & [528.405] & 530.033 & [1892.49] & 1886.67 & 44.938 & 0.1531 & {$[+0.31]$} & {$[+5.81]$} & {$[-1.628]$} & [0.0106] & {$\left[f_{43}\right]$} \\
\hline 2 & -14 & $\ldots$ & 492.999 & $\ldots$ & 2028.40 & 45.006 & 0.1581 & $\ldots$ & $\ldots$ & $\ldots$ & $\ldots$ & \\
\hline 2 & -23 & $\ldots$ & 313.886 & $\ldots$ & 3185.87 & 43.931 & 0.1616 & $\ldots$ & $\ldots$ & $\ldots$ & $\ldots$ & \\
\hline 2 & -24 & [304.349] & 304.368 & [3285.71] & 3285.49 & 43.927 & 0.1618 & {$[+0.01]$} & {$[+0.21]$} & {$[-0.020]$} & {$[0.0128]$} & {$\left[f_{40}\right]$} \\
\hline 2 & -25 & $\ldots$ & 292.768 & & 3415.67 & 43.980 & 0.1626 & & $\ldots$ & & L & \\
\hline 2 & -26 & [281.177] & 280.625 & [3556.47] & 3563.47 & 43.672 & 0.1613 & {$[-0.20]$} & {$[-7.00]$} & {$[+0.552]$} & [0.0134] & {$\left[f_{39}\right]$} \\
\hline 2 & -27 & 270.238 & 271.694 & 3700.45 & 3680.61 & 43.727 & 0.1623 & +0.54 & +19.84 & -1.456 & 0.2322 & $f_{2}$ \\
\hline 2 & -28 & $\ldots$ & 260.610 & & 3837.16 & 43.898 & 0.1637 & $\ldots$ & $\ldots$ & $\ldots$ & $\ldots$ & \\
\hline 2 & -29 & 251.274 & 251.676 & 3979.72 & 3973.36 & 43.525 & 0.1622 & +0.16 & +6.36 & -0.402 & 0.0642 & $f_{10}$ \\
\hline 2 & -30 & $\ldots$ & 245.186 & $\ldots$ & 4078.54 & 43.517 & 0.1626 & $\ldots$ & $\ldots$ & $\ldots$ & $\ldots$ & \\
\hline 2 & -31 & 235.114 & 237.167 & 4253.26 & 4216.44 & 43.613 & 0.1636 & +0.87 & +36.82 & -2.053 & 0.0545 & $f_{14}$ \\
\hline 2 & -32 & $\ldots$ & 230.026 & $\ldots$ & 4347.33 & 43.343 & 0.1629 & $\ldots$ & $\ldots$ & $\ldots$ & $\ldots$ & \\
\hline 2 & -33 & $\ldots$ & 223.962 & $\ldots$ & 4465.04 & 43.343 & 0.1634 & $\ldots$ & $\ldots$ & $\ldots$ & $\ldots$ & \\
\hline 2 & -34 & $\ldots$ & 217.217 & $\ldots$ & 4603.69 & 43.271 & 0.1635 & $\ldots$ & $\ldots$ & $\ldots$ & $\ldots$ & \\
\hline 2 & -35 & 210.489 & 211.624 & 4750.84 & 4725.37 & 43.130 & 0.1632 & +0.54 & +25.47 & -1.134 & 0.0368 & $f_{18}$ \\
\hline 2 & -36 & $\ldots$ & 205.682 & $\ldots$ & 4861.88 & 43.257 & 0.1641 & $\ldots$ & $\ldots$ & $\ldots$ & $\ldots$ & \\
\hline
\end{tabular}


Table 3. continued

\begin{tabular}{|c|c|c|c|c|c|c|c|c|c|c|c|c|}
\hline$l$ & $k$ & $\begin{array}{c}v_{\mathrm{obs}} \\
(\mu \mathrm{Hz})\end{array}$ & $\begin{array}{c}v_{\text {th }} \\
(\mu \mathrm{Hz})\end{array}$ & $\begin{array}{c}P_{\text {obs }} \\
(\mathrm{s})\end{array}$ & $\begin{array}{l}P_{\text {th }} \\
(\mathrm{s})\end{array}$ & $\begin{array}{l}\log E \\
\text { (erg) }\end{array}$ & $C_{k \ell}$ & $\begin{array}{c}\Delta P / P \\
(\%)\end{array}$ & $\begin{array}{l}\Delta P \\
(\mathrm{~s})\end{array}$ & $\begin{array}{c}\Delta v \\
(\mu \mathrm{Hz})\end{array}$ & $\begin{array}{c}\text { Ampl. } \\
(\%)\end{array}$ & Id. \\
\hline 2 & -37 & 200.674 & 199.846 & 4983.21 & 5003.86 & 43.055 & 0.1635 & -0.41 & -20.65 & +0.828 & 0.0845 & $\overline{f_{9}}$ \\
\hline 2 & -38 & $\ldots$ & 195.546 & $\ldots$ & 5113.88 & 43.053 & 0.1637 & $\ldots$ & $\ldots$ & $\ldots$ & $\ldots$ & \\
\hline 2 & -39 & $\ldots$ & 190.804 & $\ldots$ & 5240.97 & 43.247 & 0.1645 & $\ldots$ & $\ldots$ & $\ldots$ & $\ldots$ & \\
\hline 2 & -40 & 186.060 & 186.079 & 5374.61 & 5374.07 & 43.031 & 0.1641 & +0.01 & +0.54 & -0.019 & 0.0242 & $f_{27}$ \\
\hline 2 & -41 & $\ldots$ & 182.082 & $\ldots$ & 5492.04 & 42.944 & 0.1639 & $\ldots$ & $\ldots$ & $\ldots$ & $\ldots$ & \\
\hline 2 & -56 & 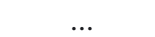 & 134.011 & & 7462.07 & 42.884 & 0.1653 & & & & & \\
\hline 2 & -57 & 131.426 & 131.703 & 7608.84 & 7592.82 & 42.918 & 0.1654 & +0.21 & +16.02 & -0.277 & 0.0278 & $f_{25}$ \\
\hline 2 & -58 & 128.284 & 129.406 & 7795.18 & 7727.60 & 42.912 & 0.1655 & +0.87 & +67.58 & -1.122 & 0.1057 & $f_{7}$ \\
\hline 2 & -59 & [127.236] & 127.284 & [7859.42] & 7856.44 & 42.832 & 0.1654 & {$[+0.04]$} & {$[+2.99]$} & {$[-0.048]$} & [0.0226] & {$\left[f_{29}\right]$} \\
\hline 2 & -60 & $\ldots$ & 125.305 & $\ldots$ & 7980.52 & 42.850 & 0.1655 & $\ldots$ & $\ldots$ & $\ldots$ & $\ldots$ & \\
\hline 4 & -11 & $\ldots$ & 1082.753 & ... & 923.57 & 45.443 & 0.0359 & & & & & \\
\hline 4 & -12 & [1020.416] & 1020.934 & [979.99] & 979.50 & 45.371 & 0.0385 & {$[+0.05]$} & {$[+0.50]$} & {$[-0.517]$} & [0.0186] & {$\left[f_{33}\right]$} \\
\hline 4 & -13 & $\ldots$ & 949.260 & $\ldots$ & 1053.45 & 44.839 & 0.0357 & $\ldots$ & $\ldots$ & $\ldots$ & $\ldots$ & \\
\hline 4 & -14 & 888.932 & 888.251 & 1124.95 & 1125.81 & 44.936 & 0.0414 & -0.08 & -0.86 & +0.681 & 0.0160 & $f_{37}$ \\
\hline 4 & -15 & $\ldots$ & 814.377 & $\ldots$ & 1227.93 & 44.376 & 0.0391 & $\ldots$ & $\ldots$ & $\ldots$ & $\ldots$ & \\
\hline 4 & -16 & 781.376 & 781.371 & 1279.79 & 1279.80 & 44.002 & 0.0360 & -0.00 & -0.01 & +0.005 & 0.0203 & $f_{32}$ \\
\hline 4 & -17 & 740.869 & 740.609 & 1349.77 & 1350.24 & 44.515 & 0.0443 & -0.04 & -0.47 & +0.260 & 0.0127 & $f_{41}$ \\
\hline 4 & -18 & $\ldots$ & 709.016 & $\ldots$ & 1410.41 & 43.998 & 0.0429 & $\ldots$ & $\ldots$ & $\ldots$ & $\ldots$ & \\
\hline 4 & -19 & $\ldots$ & 685.569 & $\ldots$ & 1458.64 & 43.526 & 0.0382 & $\ldots$ & $\ldots$ & $\ldots$ & $\ldots$ & \\
\hline 4 & -20 & [652.309] & 650.491 & [1533.02] & 1537.30 & 43.946 & 0.0449 & {$[-0.28]$} & {$[-4.29]$} & {$[+1.819]$} & [0.0107] & {$\left[f_{42}\right]$} \\
\hline 4 & -21 & $\ldots$ & 618.230 & $\ldots$ & 1617.52 & 43.666 & 0.0429 & $\ldots$ & $\ldots$ & $\ldots$ & $\ldots$ & \\
\hline 4 & -25 & $\ldots$ & 530.467 & $\ldots$ & 1885.13 & 44.014 & 0.0463 & $\ldots$ & $\ldots$ & $\ldots$ & $\ldots$ & \\
\hline 4 & -26 & 509.935 & 508.793 & 1961.04 & 1965.44 & 43.693 & 0.0449 & -0.22 & -4.40 & +1.142 & 0.0367 & $f_{19}$ \\
\hline 4 & -27 & $\ldots$ & 493.053 & $\ldots$ & 2028.18 & 43.692 & 0.0455 & $\ldots$ & $\ldots$ & $\ldots$ & $\ldots$ & \\
\hline 4 & -56 & $\ldots$ & 244.233 & 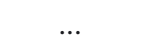 & 4094.46 & 42.883 & 0.0487 & & & & & \\
\hline 4 & -57 & [239.707] & 240.043 & [4171.76] & 4165.92 & 42.916 & 0.0488 & {$[+0.14]$} & {$[+5.84]$} & {$[-0.336]$} & [0.0167] & {$\left[f_{35}\right]$} \\
\hline 4 & -58 & $\ldots$ & 235.857 & $\ldots$ & 4239.86 & 42.914 & 0.0489 & $\ldots$ & $\ldots$ & $\ldots$ & $\ldots$ & \\
\hline 4 & -63 & $\ldots$ & 217.695 & $\ldots$ & 4593.57 & 42.778 & 0.0489 & & $\ldots$ & & $\ldots$ & \\
\hline 4 & -64 & [213.178] & 214.289 & [4690.92] & 4666.60 & 42.832 & 0.0490 & {$[+0.52]$} & {$[+24.32]$} & {$[-1.111]$} & [0.0170] & {$\left[f_{34}\right]$} \\
\hline 4 & -65 & $\ldots$ & 210.944 & $\ldots$ & 4740.60 & 42.837 & 0.0491 & $\ldots$ & $\ldots$ & $\ldots$ & $\ldots$ & \\
\hline
\end{tabular}


S. Charpinet et al.: Deep asteroseismic sounding of the sdB pulsator KIC02697388 from Kepler time series photometry

Table 4. Mode identification and details of the frequency fit obtained from the optimal model solution 2.

\begin{tabular}{|c|c|c|c|c|c|c|c|c|c|c|c|c|}
\hline$\ell$ & $k$ & $\begin{array}{c}v_{\mathrm{obs}} \\
(\mu \mathrm{Hz})\end{array}$ & $\begin{array}{c}v_{\text {th }} \\
(\mu \mathrm{Hz})\end{array}$ & $\begin{array}{c}P_{\text {obs }} \\
\text { (s) }\end{array}$ & $\begin{array}{l}P_{\text {th }} \\
(\mathrm{s})\end{array}$ & $\begin{array}{c}\log E \\
\text { (erg) }\end{array}$ & $C_{k \ell}$ & $\begin{array}{c}\Delta P / P \\
(\%)\end{array}$ & $\begin{array}{l}\Delta P \\
(\mathrm{~s})\end{array}$ & $\begin{array}{c}\Delta v \\
(\mu \mathrm{Hz})\end{array}$ & $\begin{array}{c}\text { Ampl. } \\
(\%)\end{array}$ & Id. \\
\hline 0 & 2 & & 4636.551 & & 215.68 & 41.159 & $\ldots$ & & & & & \\
\hline 0 & 1 & 3805.944 & 3810.566 & 262.75 & 262.43 & 41.536 & $\ldots$ & +0.12 & +0.32 & -4.623 & 0.0156 & $f_{38}$ \\
\hline 0 & 0 & $\ldots$ & 3089.854 & $\ldots$ & 323.64 & 42.735 & $\ldots$ & $\ldots$ & $\ldots$ & $\ldots$ & $\ldots$ & \\
\hline 1 & -6 & $\ldots$ & 565.867 & $\ldots$ & 1767.20 & 46.450 & 0.4725 & & $\ldots$ & & $\ldots$ & \\
\hline 1 & -7 & [528.405] & 530.344 & [1892.49] & 1885.57 & 45.684 & 0.4622 & {$[+0.37]$} & {$[+6.92]$} & {$[-1.939]$} & {$[0.0106]$} & {$\left[f_{43}\right]$} \\
\hline 1 & -8 & $\ldots$ & 492.235 & $\ldots$ & 2031.55 & 46.690 & 0.4737 & $\ldots$ & $\ldots$ & $\ldots$ & $\ldots$ & \\
\hline 1 & -12 & & 330.265 & & 3027.87 & 45.368 & 0.4891 & & & & & \\
\hline 1 & -13 & [304.349] & 304.303 & [3285.71] & 3286.20 & 44.856 & 0.4874 & {$[-0.01]$} & {$[-0.49]$} & {$[+0.046]$} & {$[0.0128]$} & {$\left[f_{40}\right]$} \\
\hline 1 & -14 & 284.310 & 284.998 & 3517.28 & 3508.80 & 44.870 & 0.4912 & +0.24 & +8.49 & -0.688 & 0.0622 & $f_{11}$ \\
\hline 1 & -15 & 266.164 & 262.812 & 3757.09 & 3804.99 & 44.359 & 0.4892 & -1.28 & -47.91 & +3.351 & 0.0553 & $f_{13}$ \\
\hline 1 & -16 & 251.274 & 251.961 & 3979.72 & 3968.87 & 43.977 & 0.4864 & +0.27 & +10.85 & -0.687 & 0.0642 & $f_{10}$ \\
\hline 1 & -17 & $\ldots$ & 237.875 & $\ldots$ & 4203.88 & 44.376 & 0.4930 & & $\ldots$ & & $\ldots$ & \\
\hline 1 & -18 & {$[227.150]$} & 225.806 & [4402.38] & 4428.59 & 43.850 & 0.4901 & {$[-0.60]$} & {$[-26.21]$} & {$[+1.344]$} & {$[0.0163]$} & {$\left[f_{36}\right]$} \\
\hline 1 & -19 & 220.457 & 219.735 & 4536.03 & 4550.94 & 43.547 & 0.4881 & -0.33 & -14.91 & +0.722 & 0.0333 & $f_{23}$ \\
\hline 1 & -20 & 210.489 & 209.994 & 4750.84 & 4762.04 & 44.079 & 0.4940 & -0.24 & -11.20 & +0.495 & 0.0368 & $f_{18}$ \\
\hline 1 & -21 & $\ldots$ & 199.095 & $\ldots$ & 5022.74 & 43.779 & 0.4935 & $\ldots$ & $\ldots$ & $\ldots$ & $\ldots$ & \\
\hline 1 & -22 & $\ldots$ & 192.352 & $\ldots$ & 5198.81 & 43.670 & 0.4928 & $\ldots$ & $\ldots$ & $\ldots$ & $\ldots$ & \\
\hline 1 & -23 & $\ldots$ & 183.906 & $\ldots$ & 5437.55 & 43.963 & 0.4953 & $\ldots$ & $\ldots$ & & $\ldots$ & \\
\hline 1 & -24 & 176.060 & 176.368 & 5679.88 & 5669.95 & 43.804 & 0.4950 & +0.17 & +9.93 & -0.308 & 0.0342 & $f_{20}$ \\
\hline 1 & -25 & 168.868 & 170.061 & 5921.78 & 5880.24 & 43.855 & 0.4957 & +0.70 & +41.54 & -1.193 & 0.2539 & $f_{1}$ \\
\hline 1 & -26 & [162.482] & 162.956 & [6154.54] & 6136.62 & 43.784 & 0.4957 & {$[+0.29]$} & {$[+17.92]$} & {$[-0.474]$} & {$[0.0205]$} & {$\left[f_{31}\right]$} \\
\hline 1 & -27 & 156.163 & 157.467 & 6403.57 & 6350.54 & 43.550 & 0.4948 & +0.83 & +53.02 & -1.304 & 0.1440 & $f_{5}$ \\
\hline 1 & -28 & 150.929 & 151.917 & 6625.65 & 6582.53 & 43.780 & 0.4968 & +0.65 & +43.11 & -0.989 & 0.1149 & $f_{6}$ \\
\hline 1 & -29 & 145.023 & 145.745 & 6895.45 & 6861.30 & 43.591 & 0.4964 & +0.50 & +34.14 & -0.722 & 0.1652 & $f_{3}$ \\
\hline 1 & -30 & & 141.540 & & 7065.16 & 43.300 & 0.4950 & & 列 & & & \\
\hline 1 & -31 & 137.073 & 137.309 & 7295.39 & 7282.86 & 43.534 & 0.4966 & +0.17 & +12.53 & -0.236 & 0.0340 & $f_{21}$ \\
\hline 1 & -32 & $\ldots$ & 133.067 & $\ldots$ & 7515.00 & 43.459 & 0.4964 & $\ldots$ & $\ldots$ & $\ldots$ & $\ldots$ & \\
\hline 1 & -33 & $\ldots$ & 129.804 & $\ldots$ & 7703.94 & 43.212 & 0.4958 & $\ldots$ & $\ldots$ & $\ldots$ & $\ldots$ & \\
\hline 1 & -34 & [127.236] & 126.563 & [7859.42] & 7901.22 & 43.221 & 0.4964 & {$[-0.53]$} & {$[-41.80]$} & {$[+0.673]$} & {$[0.0226]$} & {$\left[f_{29}\right]$} \\
\hline 1 & -35 & $\ldots$ & 122.957 & $\ldots$ & 8132.91 & 43.158 & 0.4968 & $\ldots$ & $\ldots$ & $\ldots$ & $\ldots$ & \\
\hline 1 & -36 & 119.546 & 119.678 & 8364.96 & 8355.76 & 43.094 & 0.4969 & +0.11 & +9.20 & -0.132 & 0.0291 & $f_{24}$ \\
\hline 1 & -37 & $\ldots$ & 116.365 & $\ldots$ & 8593.66 & 43.062 & 0.4971 & $\ldots$ & $\ldots$ & $\ldots$ & $\ldots$ & \\
\hline 1 & -38 & 113.647 & 113.428 & 8799.15 & 8816.19 & 42.972 & 0.4969 & -0.19 & -17.04 & +0.220 & 0.1489 & $f_{4}$ \\
\hline 1 & -39 & 111.481 & 110.598 & 8970.10 & 9041.75 & 43.069 & 0.4976 & -0.80 & -71.66 & +0.883 & 0.0444 & $f_{16}$ \\
\hline 1 & -40 & $\ldots$ & 107.729 & $\ldots$ & 9282.53 & 43.004 & 0.4975 & $\ldots$ & $\ldots$ & $\ldots$ & $\ldots$ & \\
\hline 1 & -43 & $\ldots$ & 100.474 & $\ldots$ & 9952.87 & 42.956 & 0.4977 & $\ldots$ & $\ldots$ & $\ldots$ & $\ldots$ & \\
\hline 1 & -44 & 97.565 & 98.362 & 10249.60 & 10166.55 & 42.952 & 0.4978 & +0.81 & +83.05 & -0.797 & 0.0276 & $f_{26}$ \\
\hline 1 & -45 & $\ldots$ & 96.185 & $\ldots$ & 10396.58 & 43.011 & 0.4980 & $\ldots$ & $\ldots$ & $\ldots$ & $\ldots$ & \\
\hline 1 & -48 & $\ldots$ & 90.505 & $\ldots$ & 11049.05 & 43.033 & 0.4983 & $\ldots$ & $\ldots$ & $\ldots$ & $\ldots$ & \\
\hline 1 & -49 & 89.115 & 88.735 & 11221.43 & 11269.50 & 42.904 & 0.4981 & -0.43 & -48.07 & +0.380 & 0.0405 & $f_{17}$ \\
\hline 1 & -50 & $\ldots$ & 87.061 & $\ldots$ & 11486.15 & 42.963 & 0.4984 & $\ldots$ & $\ldots$ & $\ldots$ & $\ldots$ & \\
\hline 1 & -62 & $\ldots$ & 70.364 & $\ldots$ & 14211.90 & 42.812 & 0.4989 & & $\ldots$ & & $\cdots$ & \\
\hline 1 & -63 & [69.770] & 69.235 & [14332.75] & 14443.50 & 42.788 & 0.4989 & {$[-0.77]$} & {$[-110.75]$} & {$[+0.535]$} & {$[0.0225]$} & {$\left[f_{30}\right]$} \\
\hline 1 & -64 & $\ldots$ & 68.176 & $\ldots$ & 14668.00 & 42.783 & 0.4989 & $\ldots$ & $\ldots$ & $\ldots$ & $\ldots$ & \\
\hline 2 & -19 & $\ldots$ & 378.439 & $\ldots$ & 2642.44 & 43.569 & 0.1558 & $\ldots$ & $\ldots$ & $\ldots$ & $\ldots$ & \\
\hline 2 & -20 & 362.702 & 361.921 & 2757.09 & 2763.04 & 44.092 & 0.1612 & -0.22 & -5.95 & +0.781 & 0.0337 & $f_{22}$ \\
\hline 2 & -21 & $\ldots$ & 343.660 & $\ldots$ & 2909.85 & 43.783 & 0.1605 & $\ldots$ & $\ldots$ & $\ldots$ & . & \\
\hline 2 & -22 & 332.366 & 332.082 & 3008.73 & 3011.30 & 43.673 & 0.1596 & -0.09 & -2.57 & +0.284 & 0.1023 & $f_{8}$ \\
\hline 2 & -23 & $\ldots$ & 317.661 & $\ldots$ & 3148.01 & 43.959 & 0.1622 & $\ldots$ & $\ldots$ & $\ldots$ & $\ldots$ & \\
\hline 2 & -24 & $\ldots$ & 304.753 & $\ldots$ & 3281.34 & 43.804 & 0.1618 & $\ldots$ & $\ldots$ & $\ldots$ & $\ldots$ & \\
\hline 2 & -25 & $\ldots$ & 293.930 & $\ldots$ & 3402.18 & 43.858 & 0.1625 & $\ldots$ & $\ldots$ & $\ldots$ & $\ldots$ & \\
\hline 2 & -26 & [281.177] & 281.711 & [3556.47] & 3549.73 & 43.790 & 0.1626 & {$[+0.19]$} & {$[+6.74]$} & {$[-0.534]$} & [0.0134] & {$\left[f_{39}\right]$} \\
\hline 2 & -27 & 270.238 & 272.229 & 3700.45 & 3673.38 & 43.546 & 0.1615 & +0.73 & +27.07 & -1.991 & 0.2322 & $f_{2}$ \\
\hline 2 & -28 & $\ldots$ & 262.729 & $\ldots$ & 3806.20 & 43.775 & 0.1635 & $\ldots$ & $\ldots$ & $\ldots$ & $\ldots$ & \\
\hline 2 & -29 & $\ldots$ & 252.040 & $\ldots$ & 3967.63 & 43.596 & 0.1632 & $\ldots$ & $\ldots$ & $\ldots$ & $\ldots$ & \\
\hline 2 & -30 & 242.449 & 244.713 & 4124.59 & 4086.43 & 43.296 & 0.1618 & +0.93 & +38.16 & -2.264 & 0.0473 & $f_{15}$ \\
\hline 2 & -31 & 235.114 & 237.352 & 4253.26 & 4213.15 & 43.522 & 0.1636 & +0.94 & +40.11 & -2.238 & 0.0545 & $f_{14}$ \\
\hline 2 & -32 & $\ldots$ & 229.911 & $\ldots$ & 4349.50 & 43.443 & 0.1635 & $\ldots$ & $\ldots$ & $\ldots$ & $\ldots$ & \\
\hline
\end{tabular}


Table 4. continued.

\begin{tabular}{|c|c|c|c|c|c|c|c|c|c|c|c|c|}
\hline$l$ & $k$ & $\begin{array}{c}v_{\mathrm{obs}} \\
(\mu \mathrm{Hz})\end{array}$ & $\begin{array}{c}v_{\text {th }} \\
(\mu \mathrm{Hz})\end{array}$ & $\begin{array}{c}P_{\text {obs }} \\
(\mathrm{s})\end{array}$ & $\begin{array}{l}P_{\text {th }} \\
(\mathrm{s})\end{array}$ & $\begin{array}{l}\log E \\
\text { (erg) }\end{array}$ & $C_{k \ell}$ & $\begin{array}{c}\Delta P / P \\
(\%)\end{array}$ & $\begin{array}{l}\Delta P \\
(\mathrm{~s})\end{array}$ & $\begin{array}{c}\Delta v \\
(\mu \mathrm{Hz})\end{array}$ & $\begin{array}{c}\text { Ampl. } \\
(\%)\end{array}$ & Id. \\
\hline 2 & -33 & $\ldots$ & 224.310 & $\ldots$ & 4458.11 & 43.216 & 0.1628 & $\ldots$ & $\ldots$ & $\ldots$ & $\ldots$ & \\
\hline 2 & -34 & $\ldots$ & 218.798 & $\ldots$ & 4570.43 & 43.227 & 0.1633 & $\ldots$ & $\ldots$ & $\ldots$ & $\ldots$ & \\
\hline 2 & -35 & [213.178] & 212.669 & [4690.92] & 4702.15 & 43.160 & 0.1636 & {$[-0.24]$} & {$[-11.23]$} & {$[+0.509]$} & {$[0.0170]$} & {$\left[f_{34}\right]$} \\
\hline 2 & -36 & $\ldots$ & 207.041 & $\ldots$ & 4829.97 & 43.096 & 0.1637 & $\ldots$ & $\ldots$ & $\ldots$ & (1) & \\
\hline 2 & -37 & 200.674 & 201.329 & 4983.21 & 4967.00 & 43.064 & 0.1638 & +0.33 & +16.22 & -0.655 & 0.0845 & $f_{9}$ \\
\hline 2 & -38 & $\ldots$ & 196.257 & $\ldots$ & 5095.36 & 42.971 & 0.1637 & $\ldots$ & $\ldots$ & $\ldots$ & $\ldots$ & \\
\hline 2 & -39 & $\ldots$ & 191.374 & $\ldots$ & 5225.36 & 43.068 & 0.1643 & $\ldots$ & $\ldots$ & $\ldots$ & & \\
\hline 2 & -40 & 186.060 & 186.409 & 5374.61 & 5364.54 & 43.005 & 0.1643 & +0.19 & +10.07 & -0.349 & 0.0242 & $f_{27}$ \\
\hline 2 & -41 & 181.614 & 182.261 & 5506.18 & 5486.64 & 42.924 & 0.1641 & +0.35 & +19.53 & -0.647 & 0.0616 & $f_{12}$ \\
\hline 2 & -42 & $\ldots$ & 178.037 & $\ldots$ & 5616.82 & 43.025 & 0.1646 & $\ldots$ & $\cdots$ & $\ldots$ & $\ldots$ & \\
\hline 2 & -52 & $\ldots$ & 144.706 & $\ldots$ & 6910.57 & 42.870 & 0.1651 & $\ldots$ & $\ldots$ & $\ldots$ & $\ldots$ & \\
\hline 2 & -53 & 141.994 & 142.001 & 7042.57 & 7042.22 & 42.933 & 0.1653 & +0.01 & +0.35 & -0.007 & 0.0227 & $f_{28}$ \\
\hline 2 & -54 & $\ldots$ & 139.220 & $\ldots$ & 7182.88 & 42.897 & 0.1653 & $\ldots$ & $\ldots$ & $\ldots$ & $\ldots$ & \\
\hline 2 & -55 & $\ldots$ & 136.802 & $\ldots$ & 7309.85 & 42.840 & 0.1653 & $\ldots$ & $\ldots$ & $\ldots$ & $\ldots$ & \\
\hline 2 & -56 & $\ldots$ & 134.385 & $\ldots$ & 7441.33 & 42.917 & 0.1655 & $\ldots$ & $\ldots$ & $\ldots$ & $\ldots$ & \\
\hline 2 & -57 & 131.426 & 132.018 & 7608.84 & 7574.74 & 42.837 & 0.1654 & +0.45 & +34.10 & -0.592 & 0.0278 & $f_{25}$ \\
\hline 2 & -58 & $\ldots$ & 129.958 & $\cdots$ & 7694.77 & 42.793 & 0.1653 & $\ldots$ & $\ldots$ & $\ldots$ & $\ldots$ & \\
\hline 2 & -59 & 128.284 & 127.827 & 7795.18 & 7823.09 & 42.878 & 0.1655 & -0.36 & -27.91 & +0.458 & 0.1057 & $f_{7}$ \\
\hline 2 & -60 & $\ldots$ & 125.688 & $\ldots$ & 7956.19 & 42.802 & 0.1655 & $\ldots$ & $\ldots$ & $\cdots$ & $\ldots$ & \\
\hline 4 & -11 & $\ldots$ & 1101.764 & $\ldots$ & 907.64 & 45.068 & 0.0305 & $\ldots$ & & & & \\
\hline 4 & -12 & [1020.416] & 1020.830 & [979.99] & 979.60 & 45.198 & 0.0381 & {$[+0.04]$} & {$[+0.40]$} & {$[-0.413]$} & [0.0186] & {$\left[f_{33}\right]$} \\
\hline 4 & -13 & $\ldots$ & 941.344 & $\ldots$ & 1062.31 & 44.747 & 0.0367 & $\ldots$ & $\ldots$ & $\ldots$ & & \\
\hline 4 & -14 & 888.932 & 886.446 & 1124.95 & 1128.10 & 44.768 & 0.0411 & -0.28 & -3.15 & +2.486 & 0.0160 & $f_{37}$ \\
\hline 4 & -15 & $\ldots$ & 817.298 & $\ldots$ & 1223.54 & 44.415 & 0.0412 & $\ldots$ & $\ldots$ & $\ldots$ & $\ldots$ & \\
\hline 4 & -16 & 781.376 & 781.218 & 1279.79 & 1280.05 & 43.821 & 0.0347 & -0.02 & -0.26 & +0.158 & 0.0203 & $f_{32}$ \\
\hline 4 & -17 & 740.869 & 740.142 & 1349.77 & 1351.09 & 44.244 & 0.0444 & -0.10 & -1.33 & +0.727 & 0.0127 & $f_{41}$ \\
\hline 4 & -18 & $\ldots$ & 698.755 & $\ldots$ & 1431.12 & 43.691 & 0.0413 & $\ldots$ & $\ldots$ & $\ldots$ & $\ldots$ & \\
\hline 4 & -19 & $\ldots$ & 680.138 & $\ldots$ & 1470.29 & 43.561 & 0.0403 & $\ldots$ & $\ldots$ & $\ldots$ & $\ldots$ & \\
\hline 4 & -20 & [652.309] & 651.197 & [1533.02] & 1535.63 & 44.101 & 0.0451 & {$[-0.17]$} & {$[-2.62]$} & {$[+1.112]$} & [0.0107] & {$\left[f_{42}\right]$} \\
\hline 4 & -21 & $\ldots$ & 620.607 & $\ldots$ & 1611.33 & 43.809 & 0.0442 & $\ldots$ & $\ldots$ & $\ldots$ & $\ldots$ & \\
\hline 4 & -25 & $\ldots$ & 533.086 & $\ldots$ & 1875.87 & 43.863 & 0.0460 & $\cdots$ & $\ldots$ & $\cdots$ & & \\
\hline 4 & -26 & 509.935 & 511.286 & 1961.04 & 1955.85 & 43.818 & 0.0462 & +0.26 & +5.18 & -1.352 & 0.0367 & $f_{19}$ \\
\hline 4 & -27 & $\ldots$ & 493.963 & $\ldots$ & 2024.44 & 43.534 & 0.0448 & $\cdots$ & $\cdots$ & $\ldots$ & $\ldots$ & \\
\hline 4 & -56 & $\ldots$ & 244.911 & 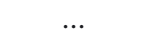 & 4083.11 & 42.915 & 0.0488 & $\ldots$ & & & & \\
\hline 4 & -57 & [239.707] & 240.578 & [4171.76] & 4156.66 & 42.837 & 0.0488 & {$[+0.36]$} & {$[+15.10]$} & {$[-0.871]$} & [0.0167] & {$\left[f_{35}\right]$} \\
\hline 4 & -58 & $\ldots$ & 236.820 & $\ldots$ & 4222.61 & 42.792 & 0.0487 & $\ldots$ & $\ldots$ & $\ldots$ & $\ldots$ & \\
\hline
\end{tabular}

